Bull. Chem. Soc. Ethiop. 2020, 34(2), 385-405.

ISSN 1011-3924

(C) 2020 Chemical Society of Ethiopia and The Authors

Printed in Ethiopia

DOI: https://dx.doi.org/10.4314/bcse.v34i2.15

\title{
USES OF $\beta$-DIKETONES FOR THE SYNTHESIS OF NOVEL HETEROCYCLIC COMPOUNDS AND THEIR ANTITUMOR EVALUATIONS
}

\author{
Rafat M. Mohareb ${ }^{1 *}$, Mona M. Kamel' ${ }^{2}$ and Yara R. Milad ${ }^{2,3}$ \\ ${ }^{1}$ Department of Chemistry, Faculty of Science, Cairo University, Giza, Egypt \\ ${ }^{2}$ Department of Pharmaceutical Organic Chemistry, Faculty of Pharmacy, Cairo University, \\ P.O. BOX 11562, Cairo, Egypt \\ ${ }^{3}$ Jean Coutu, 531 Jarry Est, Montreal Quebec, Canada
}

(Received May 28, 2020; Revised October 10, 2020; Accepted October 20, 2020)

\begin{abstract}
The reaction of the 3-oxo-N,3-diphenylpropan-amide (3) with either malononitrile or ethyl cyanoacetate in ammonium acetate gave the 1,2-dihydropyridine derivatives $6 \mathbf{a}$ or $\mathbf{6 b}$, respectively. On the other hand, carrying the same reaction in the presence of triethylamine gave the 1,6-dihydropyridine derivatives 7a and $\mathbf{7 b}$, respectively. Moreover, compound 3 reacted with 2-aminoprop-1-ene-1,1,3-tricarbonitrile to give the pyridine derivative 9. Compound $\mathbf{7 b}$ reacted with the active methylene derivatives $\mathbf{1 0 a}, \mathbf{b}$ and $\mathbf{4 a}, \mathbf{b}$ to give the naphthyridine derivatives 11a,b and 12a,b; respectively. Compound $\mathbf{3}$ was also used for the synthesis of thiophene derivatives 13a,b and 16a,b. In addition, the reaction of ethyl benzoylacetate (1) with $o$-phenylene diamine gave the benzimidazole derivative 18. The reactivity of the latter product towards different reagents was studied to give different products. The cytotoxicity of the newly synthesized products was studied towards some cancer and normal cell lines, in addition toxicity of compounds was measured and docking of the most active compounds was done. Compounds $6 \mathbf{b}, 7 \mathbf{b}, 9,13 a, 13 b, 16 a, 20 b, 20 c, 24 b, 25$ and $26 b$ exhibited optimal cytotoxic effect against cancer tested cell lines. These active compounds were evaluated against c-Met kinase using foretinib as the reference drug where all compounds expressed higher activity than the reference drug.
\end{abstract}

KEY WORDS: Ethyl benzoylacetate, Pyridine, Benzimidazole, Cytotoxicity

\section{INTRODUCTION}

It is of great interest to note that pyridine and pyrimidine derivatives were one of the most classes of compounds due to their high pharmaceutical and biological values. Especially fused pyridine derivatives have varieties of biological uses. Among such activities, they are known to exhibit high pharmacological, CNS depressant [1, 2], neuroleptic [3] and tuberculostatic [4] activities. In addition, a largenumber of pyridines were used as antimicrobial agents [5], inhibitors of glycogen syntheses kinase-3 (GSK-3) [6] and potent antitumor agents [7]. Moreover, benzimidazoles represent a group of compounds with a large number of pharmaceutical applications and many of them were basic nucleus of drugs structures. Some of them were naturally occurring nucleotides that capable to interact with biopolymers to enhance better biological properties. It was noticed that some 2-aminobenzimidazoles showed interesting antimicrobial effect, especially, their corresponding carbamate derivatives were obtained in good yields and exhibited significant in vivo antifilarial activity [8]. Within the field of drug designing such group of compounds have a great affinity towards different enzymes and protein receptors [9]. Optimization of benzimidazole-based structures has resulted in marketed drugs, e.g. within the field of chemotherapy it was found that omeprazole [10] and pimobendan [11] they were found not only as good therapeutically active drugs but also for treatment of heart feeler problems. Moreover, a large number of benzimidazoles derivatives are well known for their antimicrobial [12-15], anthelmintic [16], antiviral [17-18] and antifungal [19-21] activities. Many benzimidazole containing compounds are known as anticancer agents [22-28]. Within the field of topoisomerase inhibitors it was found that some benzimidazole was used as

*Corresponding author. E-mail: raafat_mohareb@yahoo.com

This work is licensed under the Creative Commons Attribution 4.0 International License 
topoisomerase inhibitory drugs, e.g. Hoechst 33258 and Hoechst 33342 (Figure 1) [29, 30]. Within the field of DNA binders, it was also found that bis-benzimidazole derivatiives are used as head to head binders [31]. Within the field of cancer chemotherapy, many drugs containing benzimidazole nucleus are known through the market like RAF265 (CHIR-265; Novartis Pharmaceuticals, Basel, Switzerland) and AZD6244 (ARRY-142886; AstraZeneca, London, England). A very active known drug is RAF265 resulted in a reduction in tumor cell growth and in tumor cell apoptosis [32].

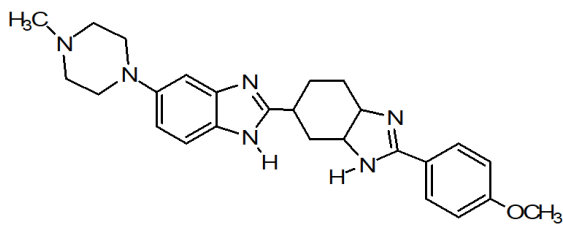

Hoechst 33342

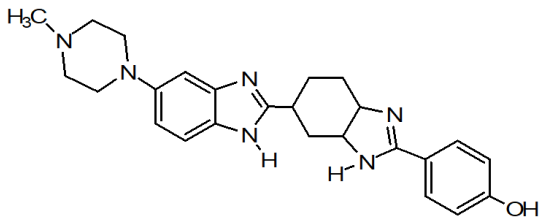

Hoechst 33258

Figure 1. Examples of topoisomerase inhibitors containing benzimidazole nucleus.

Due to such high importance of pyridine and benzimidazole derivatives was focused on the efficient synthesis of new pyridine and benzimidazole derivatives starting from ethyl benzoylacetate followed by their cytotoxic evaluations against human cancer and normal cell lines.

\section{EXPERIMENTAL}

${ }^{13} \mathrm{C}$ NMR and ${ }^{1} \mathrm{H}$ NMR spectra were recorded on Bruker DPX200 instrument in $\mathrm{CDCl}_{3}$ and DMSO with TMS as internal standard for protons and solvent signals as internal standard for carbon spectra. Chemical shift values are mentioned in $\delta(\mathrm{ppm})$. Mass spectra were recorded on EIMS (Shimadzu) and ESI-esquire 3000 Bruker Daltonics instrument. Elemental analyses were carried out by the Microanalytical Data Unit at Ludwig-Maximilians-Universität-München, Germany. The progress of all reactions was monitored by TLC on $2 \times 5 \mathrm{~cm}$ pre-coated silica gel 60 F254 plates of thickness of $0.25 \mathrm{~mm}$ (Merck).

General procedure for the synthesis of the 1,2-dihydropyridine derivatives $\mathbf{6} \boldsymbol{a}, \boldsymbol{b}$

Either of malononitrile $(0.66 \mathrm{~g}, 0.01 \mathrm{~mol})$ or ethyl cyanoacetate $(1.13 \mathrm{~g}, 0.01 \mathrm{~mol})$ together with ammonium acetate $(0.50 \mathrm{~g})$ were added to a dry solid of compound $3(2.16 \mathrm{~g}, 0.01 \mathrm{~mol})$. The reaction mixture, in each case, was heated in an oil bath at $120{ }^{\circ} \mathrm{C}$ for $15 \mathrm{~min}$. The solid product formed after boiling with ethanol was collected by filtration.

6-Hydroxy-2-imino-1,4-diphenyl-1,2-dihydropyridine-3-carbonitrile (6a). Yellow crystals $(\mathrm{EtOH})$, yield $74 \%$ (2.12 g), mp 119-121 ${ }^{\circ} \mathrm{C}$. IR $v_{\max } \mathrm{cm}^{-1}: 3544-3260,3056,2957,2207,1688$, 1662, $1604(\mathrm{C}=\mathrm{C}) .{ }^{1} \mathrm{H}$ NMR $\left(\mathrm{DMSO}_{6} 400 \mathrm{MHz}\right): \delta=4.15\left(\mathrm{~s}, 2 \mathrm{H}, \mathrm{CH}_{2}\right), 7.30-7.99(\mathrm{~m}, 10 \mathrm{H}$, $\left.2 \mathrm{C}_{6} \mathrm{H}_{5}\right), 10.21(\mathrm{~s}, 1 \mathrm{H}, \mathrm{NH}) .{ }^{13} \mathrm{C}$ NMR (DMSO-d $675 \mathrm{MHz}$ ): $\delta=49.5$ (pyridine C-3), 103.6, $104.5(\mathrm{C}=\mathrm{C}), 119.5(\mathrm{CN}), 120.19,121.3,123.9,124.1,124.8,125.2,125.5,126.0,134.5,136.8$ (two benzene, pyridine $\mathrm{C}$ ), $165.9(\mathrm{C}=\mathrm{O}), 175.2(\mathrm{C}=\mathrm{N})$. EIMS m/z $287[\mathrm{M}]^{+}(20)$; anal. calcd. for $\mathrm{C}_{18} \mathrm{H}_{13} \mathrm{~N}_{3} \mathrm{O}$ (287.11): C, 75.25; H, 4.56; N, 14.63. Found: C, 72.06; H, 4.72; N, 14.88 .

Ethyl 6-hydroxy-2-imino-1,4-diphenyl-1,2-dihydropyridine-3-carboxylate (6b). Yellow crystals (EtOH), yield 70\% (2.33 g), mp 277-279 ${ }^{\circ} \mathrm{C}$. IR $v_{\max } \mathrm{cm}^{-1}: 3443-3259,3092,2959,2888,1695$, 1689, 1636. ${ }^{1} \mathrm{H}$ NMR (DMSO-d 6 $400 \mathrm{MHz}$ ): $\delta=1.14\left(\mathrm{t}, 3 \mathrm{H}, \mathrm{J}=7.03 \mathrm{~Hz}, \mathrm{OCH}_{2} \underline{\mathrm{CH}}_{3}\right.$ ), 4,18 (q, 
$\left.2 \mathrm{H}, \mathrm{J}=7.03 \mathrm{~Hz}, \mathrm{OCH}_{2} \mathrm{CH}_{3}\right), 5.95\left(\mathrm{~s}, 2 \mathrm{H}, \mathrm{CH}_{2}\right), 7.30-7.99\left(\mathrm{~m}, 10 \mathrm{H}, 2 \mathrm{C}_{6} \mathrm{H}_{5}\right), 10.21\left(\mathrm{~s}, 1 \mathrm{H}, \mathrm{D}_{2} \mathrm{O}\right.$ exchangeable, $\mathrm{NH}) .{ }^{13} \mathrm{C}$ NMR (DMSO-d $\left.675 \mathrm{MHz}\right) \delta: 13.6\left(\mathrm{OCH}_{2} \underline{\mathrm{CH}}_{3}\right), 50.3\left(\mathrm{OCH}_{2} \mathrm{CH}_{3}\right), 50.6$ (pyridine C-3), 103.8, $104.9(\mathrm{C}=\mathrm{C}), 116.5(\mathrm{CN}), 119.0,120.5,122.5,123.6,124.4,124.6,125.1$, 125.7 (two benzene C), $163.8(\mathrm{C}=\mathrm{N}), 165.1,166.4(2 \mathrm{C}=\mathrm{O})$, EIMS m/z $334[\mathrm{M}]^{+}$(28). Anal. calcd. for $\mathrm{C}_{20} \mathrm{H}_{18} \mathrm{~N}_{2} \mathrm{O}_{3}$ (334.38): C, 71.84; H, 5.43; N, $8.38 \%$. Found: C, 71.69; H, 5.51; N, 8.49.

General procedure for the synthesis of the 1,6-dihydopyridine-2-carboxylate 7a,b

Either malononitrile $(0.66 \mathrm{~g}, 0.01 \mathrm{~mol})$ or ethyl cyanoacetate $(1.13 \mathrm{~g}, 0.01 \mathrm{~mol})$ was added to a solution of compound $3(2.16 \mathrm{~g}, 0.01 \mathrm{~mol})$ in 1,4-dioxane $(40 \mathrm{~mL})$ containing triethylamine $(0.50 \mathrm{~mL})$. The reaction mixture, in each case was heated under reflux for $3 \mathrm{~h}$ then evaporated under vacuum. The solid product produced after triturating the remaining product with diethyl ether was collected by filtration.

4-Amino-5-benzoyl-6-oxo-1-phenyl-1,6-dihydropyridine-2-carbonitrile (7a). Pale yellow crystals (1,4-dioxane), yield $80 \%$ (2.12 g), mp 160-168 ${ }^{\circ} \mathrm{C}$. IR $v_{\max } \mathrm{cm}^{-1}: 3544-3260,3058$, 2957, 1705, 1689, 1632. ${ }^{1} \mathrm{H}$ NMR (DMSO-d $\left.{ }_{6} 400 \mathrm{MHz}\right): \delta=4.28,5.31\left(2 \mathrm{~s}, 4 \mathrm{H}, \mathrm{D}_{2} \mathrm{O}\right.$ exchangeable, $\left.2 \mathrm{NH}_{2}\right), 6.15(\mathrm{~s}, 1 \mathrm{H}$, pyridine $\mathrm{H}-4), 7.31-7.68\left(\mathrm{~m}, 10 \mathrm{H}, 2 \mathrm{C}_{6} \mathrm{H}_{5}\right) .{ }^{13} \mathrm{C} \mathrm{NMR}$ $\left(\right.$ DMSO-d $_{6} 75$ MHz): $\delta=119.5,120.1,120.7,122.0,123.6,124.7,125.6,127.3,129.8,130.2$, 131.6, 139.4 (two benzene, pyridine C), 164.3, 166.4 (2 C=O). EIMS m/z $305[\mathrm{M}]^{+}$(26). Anal. calcd. for $\mathrm{C}_{18} \mathrm{H}_{15} \mathrm{~N}_{3} \mathrm{O}_{2}$ (305.54): C, 70.81; H, 4.95; N, 13.76.

Ethyl 4-amino-5-benzoyl-6-oxo-1-phenyl-1,6-dihydropyridine-2-carboxylate (7b). Yellow crystals (1,4-dioxane), yield $81 \%(2.47 \mathrm{~g}), \mathrm{mp} 177-179{ }^{\circ} \mathrm{C}$. IR $v_{\max } \mathrm{cm}^{-1}: 3540-3320,3065$, 2955, 2889, 1718, 1688, 1633. ${ }^{1} \mathrm{H}$ NMR (DMSO-d $\left.{ }_{6} 400 \mathrm{MHz}\right): \delta=4.25\left(\mathrm{~s}, 2 \mathrm{H}, \mathrm{D}_{2} \mathrm{O}\right.$ exchangeable, $\left.\mathrm{NH}_{2}\right), 6.11(\mathrm{~s}, 1 \mathrm{H}$, pyridine $\mathrm{H}-4), 7.28-7.67\left(\mathrm{~m}, 10 \mathrm{H}, 2 \mathrm{C}_{6} \mathrm{H}_{5}\right), 11.42\left(\mathrm{~s}, 1 \mathrm{H}, \mathrm{D}_{2} \mathrm{O}\right.$ exchangeable, OH). ${ }^{13} \mathrm{C}$ NMR (DMSO-d $\left.675 \mathrm{MHz}\right): \delta=119.6,120.2,120.9,122.5,123.8$, 124.3, 124.6, 125.7, 129.8, 130.2, 133.2, 139.4 (two benzene, pyridine C), 164.2, 166.8 (2 $\mathrm{C}=\mathrm{O}$ ). EIMS m/z $306.32[\mathrm{M}]^{+}$(28). Anal. calcd. for $\mathrm{C}_{18} \mathrm{H}_{14} \mathrm{~N}_{2} \mathrm{O}_{3}$ (306.32): C, 70.58; H, 4.61; N, 9.15. Found: $\mathrm{C}, 70.80 ; \mathrm{H}, 4.88 ; \mathrm{N}, 9.05$.

\section{2,4-Diamino-7-oxo-5,8-diphenyl-7,8-dihydro-1,8-naphthyridine-3-carbonitrile (9)}

2-Aminoprop-1-ene-1,1,3-tricarbonitrile (8) (1.32 g, $0.01 \mathrm{~mol})$ was added to a solution of compound $3(2.16 \mathrm{~g}, 0.01 \mathrm{~mol})$ in 1,4-dioxane $(40 \mathrm{~mL})$ containing triethylamine $(0.50 \mathrm{~mL})$. The reaction mixture was heated under reflux for $6 \mathrm{~h}$ then evaporated under vacuum. The solid product produced after triturating the remaining product with diethyl ether was collected by filtration. Pale yellow crystals (1,4-dioxane), yield $86 \%(3.03 \mathrm{~g}), \mathrm{mp} 260-263{ }^{\circ} \mathrm{C}$. IR $v_{\max } \mathrm{cm}^{-1}$ : 3473, 3327, 3056, 2220, 1688, 1623. ${ }^{1} \mathrm{H}$ NMR (DMSO-d 6 , $\left.400 \mathrm{MHz}\right) \delta=4.92,5.68(2 \mathrm{~s}, 4 \mathrm{H}$, $\mathrm{D}_{2} \mathrm{O}$ exchangeable, $\left.2 \mathrm{NH}_{2}\right), 6.80(\mathrm{~s}, 1 \mathrm{H}$ pyridine $\mathrm{H}-6), 7.31-7.58\left(\mathrm{~m}, 10 \mathrm{H}, 2 \mathrm{C}_{6} \mathrm{H}_{5}\right) .{ }^{13} \mathrm{C} \mathrm{NMR}$ $\left(\mathrm{DMSO}_{6}, 75 \mathrm{MHz}\right): \delta=116.8(\mathrm{CN}), 120.1,121.3,122.3,123.5,124.0,124.8,125.1,126.4$, $128.1,130.2,131.2,131.8,133.2,134.2$ (two $\mathrm{C}_{6} \mathrm{H}_{5}$, two pyridine $\mathrm{C}$ ), $162.4(\mathrm{C}=\mathrm{O}), 169.5(\mathrm{C}=\mathrm{N})$. EIMS m/z $353[\mathrm{M}]^{+}$(22). Anal. calcd. for $\mathrm{C}_{21} \mathrm{H}_{15} \mathrm{~N}_{3} \mathrm{O}$ (353.39): C, 71.38; H, 4.28; N, 19.82 . Found: C, 71.42; H, 4.31; N, 19.72.

General procedure for the synthesis of the 1,6-naphthridine derivatives 11a,b

Either acetylacetone $(1.00 \mathrm{~g}, 0.01 \mathrm{~mol})$ or ethyl acetoacetate $(1.30 \mathrm{~g}, 0.01 \mathrm{~mol})$ was added to a solution of compound $7 \mathbf{b}(3.06 \mathrm{~g}, 0.01 \mathrm{~mol})$ in 1,4-dioxane $(40 \mathrm{~mL})$ containing triethylamine $(0.50 \mathrm{~mL})$. The reaction mixture was heated under reflux for $4 \mathrm{~h}$ then poured onto ice/water and the formed solid product was collected by filtration. 
8-Benzoyl-2,4-dimethyl-6-phenyl-1,6-naphthridine-5,7(1H,6H)-dione (11a). Orange crystals (1,4-dioxane) yield $76 \%(2.81 \mathrm{~g}), \mathrm{mp} 116-118{ }^{\circ} \mathrm{C}$. IR $v_{\max } \mathrm{cm}^{-1}: 3430-3261,3065,2919,1688-$ 1662. ${ }^{1} \mathrm{H}$ NMR (DMSO-d $\left.{ }_{6} 400 \mathrm{MHz}\right) \delta=2.51,4.1\left(2 \mathrm{~s}, 6 \mathrm{H}, 2 \mathrm{CH}_{3}\right), 5.95(\mathrm{~s}, 1 \mathrm{H}$, pyridine $\mathrm{H}-3)$, 7.25-7.54 (m, 10H, 2C $\left.\mathrm{C}_{6} \mathrm{H}_{5}\right), 10.16\left(\mathrm{~s}, 1 \mathrm{H}, \mathrm{D}_{2} \mathrm{O}\right.$ exchangeable, $\left.\mathrm{NH}\right) .{ }^{13} \mathrm{C}$ NMR (DMSO-d $\mathrm{d}_{6} 75$

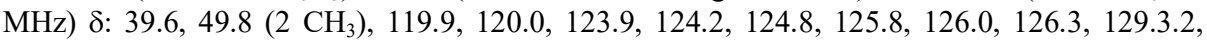
129.4, 130.6, 132.6, 134.1, 134.2 (two benzene $\mathrm{C}$, two pyridine $\mathrm{C}$ ), 165.8, 170.2, 175.2 (3 $\mathrm{C}=\mathrm{O}$ ). EIMS m/z $370[\mathrm{M}]^{+}(20)$. Anal. calcd. for $\mathrm{C}_{23} \mathrm{H}_{18} \mathrm{~N}_{2} \mathrm{O}_{3}$ (370.41): C, 74.58; H, 4.90; N, 7.56. Found: C, 74.66; H, 4.69; N, 7.38.

8-Benzoyl-4-hydroxy-2-methyl-6-phenyl-1,6-naphthridine-5,7(1H,6H)-dione (11b). Yellow crystals (1,4-dioxane), yield $80 \%$ (2.97 g), mp 97-99 ${ }^{\circ} \mathrm{C}$. IR $v_{\max } \mathrm{cm}^{-1}: 3550-3320,3055,2955$, 2889, 1720-1688, 1633. ${ }^{1} \mathrm{H}$ NMR (DMSO-d $\left.6400 \mathrm{MHz}\right) \delta=2.51\left(\mathrm{~s}, 3 \mathrm{H}, \mathrm{CH}_{3}\right), 5.45(\mathrm{~s}, 1 \mathrm{H}$, pyridine $\mathrm{H}-3), 7.01-7.31\left(\mathrm{~m}, 10 \mathrm{H}, 2 \mathrm{C}_{6} \mathrm{H}_{5}\right), 8.20\left(\mathrm{~s}, 1 \mathrm{H}, \mathrm{D}_{2} \mathrm{O}\right.$ exchangeable, $\left.\mathrm{NH}\right), 9.01(\mathrm{~s}, 1 \mathrm{H}$, $\mathrm{D}_{2} \mathrm{O}$ exchangeable, $\left.\mathrm{OH}\right) .{ }^{13} \mathrm{C}$ NMR (DMSO-d 6 $\left.75 \mathrm{MHz}\right) \delta: 25.1,\left(\mathrm{CH}_{3}\right), 118.9,119.8,120.3$, 123.6, 123.9, 124.2, 125.3, 125.6, 128.6, 129.4, 132.1, 133.6, 134.2, 134.6 (two benzene, two pyridine C), 165.6, 172.0, $180.3(3 \mathrm{C}=\mathrm{O})$. EIMS m/z $372[\mathrm{M}]^{+}(18)$. Anal. calcd. for $\mathrm{C}_{22} \mathrm{H}_{16} \mathrm{~N}_{2} \mathrm{O}_{4}$ (372.38): C, 70.96; H, 4.33; N, 7.52. Found: C, 70.82; H, 4.48; N, 7.63.

General procedure for the synthesis of the 5,6,7,8-tetrahydro-1,6-naphthyridine derivatives $12 a, b$

Either malononitrile $(0.66 \mathrm{~g}, 0.01 \mathrm{~mol})$ or ethyl cyanoacetate $(1.13 \mathrm{~g}, 0.01 \mathrm{~mol})$ was added to a solution of compound $7 \mathbf{b}(3.06 \mathrm{~g}, 0.01 \mathrm{~mol})$ in 1,4-dioxane $(40 \mathrm{~mL})$ containing triethylamine $(0.50 \mathrm{~mL})$. The reaction mixture was heated under reflux for $8 \mathrm{~h}$ then poured onto ice/water and the formed solid product was collected by filtration.

2-Amino-5,7-dioxo-4,6-diphenyl-5,6,7,8-tetrahydro-1,6-naphthyridine-3-carbonitrile (12a). Yellow crystals (1,4-dioxane), yield 88\% (3.11 g), mp 140-143 ${ }^{\circ} \mathrm{C}$. IR $v_{\max } \mathrm{cm}^{-1}$ : 3409-3334, $3055,2923,2210,1680-1662 .{ }^{1} \mathrm{H}$ NMR $\left(\mathrm{DMSO}_{-} \mathrm{d}_{6} 400 \mathrm{MHz}\right): \delta=3.58,4.20\left(2 \mathrm{~s}, 4 \mathrm{H}, \mathrm{D}_{2} \mathrm{O}\right.$ exchangeable, $\left.2 \mathrm{NH}_{2}\right), 6.20(\mathrm{~s}, 1 \mathrm{H}$, pyridine $\mathrm{H}-3), 7.20(\mathrm{~s}, 1 \mathrm{H}$, pyridine $\mathrm{H}-3), 7.27-7.54(\mathrm{~m}, 10 \mathrm{H}$, $2 \mathrm{C}_{6} \mathrm{H}_{5}$ ). ${ }^{13} \mathrm{C}$ NMR (DMSO-d 6 $75 \mathrm{MHz}$ ) $\delta$ : 48.9 (pyridine C-3), 119.9, 120.3, 120.6, 123.9, 125.8, 128.6, 129.3.2, 129.4, 132.6, 134.1, 140.4 (two benzene $\mathrm{C}$, two pyridine $\mathrm{C}$ ), 165.8, 166.3, 170.1 $(3 \mathrm{C}=\mathrm{O}), 195.3(\mathrm{C}=\mathrm{N})$. EIMS m/z 372[M] $]^{+}(20)$; anal. calcd. for $\mathrm{C}_{21} \mathrm{H}_{16} \mathrm{~N}_{4} \mathrm{O}_{3}$ (372.38): C, 67.73; H, 4.33; N, 15.05. Found: C, 67.58; H, 4.04; N, 15.25 .

2-Hydroxy-5,7-dioxo-4,6-diphenyl-5,6,7,8-tetrahydro-1,6-naphthyridine-3-carbonitrile (12b). Yellow crystals (1,4-dioxane), yield 70\% (2.48 g), mp 100-103 ${ }^{\circ} \mathrm{C}$. IR $v_{\max } \mathrm{cm}^{-1}$ : 3542-3260, 3093, 2956, 1688-1662, 1605. ${ }^{1} \mathrm{H}$ NMR (DMSO-d $\left.\mathrm{d}_{6} 400 \mathrm{MHz}\right) \delta=3.88\left(\mathrm{~s}, 2 \mathrm{H}, \mathrm{D}_{2} \mathrm{O}\right.$ exchangeable, $\left.\mathrm{NH}_{2}\right), 4.14(\mathrm{~s}, 1 \mathrm{H}$, pyridine $\mathrm{H}-3), 7.27-7.67\left(\mathrm{~m}, 11 \mathrm{H}, 2 \mathrm{C}_{6} \mathrm{H}_{5}\right.$, pyridine $\left.\mathrm{H}-3\right), 10.19$ (s, $1 \mathrm{H}, \mathrm{D}_{2} \mathrm{O}$ exchangeable, $\left.\mathrm{OH}\right) .{ }^{13} \mathrm{C}$ NMR (DMSO-d 6 $\left.75 \mathrm{MHz}\right) \delta=49.5$ (pyridine C-3), 119.8, $120.2,122.5,123.6,124.2,124.6,125.3,125.8,130.2,132.5,133.2,134.1,134.6 .135 .2$ (two benzene C, two pyridine C), 165.6, $170.1(2 \mathrm{C}=\mathrm{O}), 171.1(\mathrm{C}=\mathrm{N})$. EIMS m/z $373[\mathrm{M}]^{+}(20)$. Anal. calcd. for $\mathrm{C}_{21} \mathrm{H}_{15} \mathrm{~N}_{3} \mathrm{O}_{4}$ (373.37): C, 67.56; H, 4.05; N, 11.25. Found: C, 67.33; H, 3.87; N, 11.41.

General procedure for the synthesis of the thiophene derivatives $13 a, b$

Each of elemental sulfur $(0.32 \mathrm{~g}, 0.01 \mathrm{~mol})$ and either malononitrile $(0.66 \mathrm{~g}, 0.01 \mathrm{~mol})$ or ethyl cyanoacetate $(1.13 \mathrm{~g}, 0.01 \mathrm{~mol})$ was added to a solution of compound $3(2.16 \mathrm{~g}, 0.01 \mathrm{~mol})$ in 1,4-dioxane $(40 \mathrm{~mL})$ containing triethylamine $(0.50 \mathrm{~mL})$. The reaction mixture, in each case, was heated under reflux for $3 \mathrm{~h}$ then poured onto ice/water containing few drops of hydrochloric acid and the formed solid product was collected by filtration. 
5-Amino-4-cyano-N,3-diphenylthiophene-2-carboxamide (13a). Orange crystals (1,4-dioxane), yield 90\% (2.87 g), mp 120-122 ${ }^{\circ} \mathrm{C}$. IR $v_{\max } \mathrm{cm}^{-1}: 3439-33259,3056,2923,2211,1687 .{ }^{1} \mathrm{H}$ NMR (DMSO-d $\left.\mathrm{d}_{6} 400 \mathrm{MHz}\right): \delta=4.15\left(\mathrm{~s}, 2 \mathrm{H}, \mathrm{D}_{2} \mathrm{O}\right.$ exchangeable, $\left.\mathrm{NH}_{2}\right), 7.31-7.58(\mathrm{~m}, 10 \mathrm{H}$, $\left.2 \mathrm{C}_{6} \mathrm{H}_{5}\right), 10.21\left(\mathrm{~s}, 1 \mathrm{H}, \mathrm{D}_{2} \mathrm{O}\right.$ exchangeable, $\left.\mathrm{NH}\right),{ }^{13} \mathrm{C}$ NMR (DMSO- $\left.\mathrm{d}_{6} 75 \mathrm{MHz}\right): \delta=117.5(\mathrm{CN})$, $120.3,121.6,122.8,123.6,124.4,124.8,125.1,125.8,136.7,139.5 .140 .4,142.2$ (two benzene $\mathrm{C}$, thiophene C), $165.8(\mathrm{C}=\mathrm{O})$. EIMS m/z $319[\mathrm{M}]^{+}$(26). Anal. calcd. for $\mathrm{C}_{18} \mathrm{H}_{13} \mathrm{~N}_{3} \mathrm{OS}(319.38)$ : C, 67.69; H, 4.10; N, 13.16; S, 10.04. Found: C, 67.56; H, 3.89; N, 12.88; S, 10.38 .

Ethyl 2-amino-4-phenyl-5-(phenylcarbamoyl)thiophene-3-carboxylate (13b). Orange crystals (1,4-dioxane), yield $73 \%$ (2.67 g), mp 90-93 ${ }^{\circ} \mathrm{C}$. IR $v_{\max } \mathrm{cm}^{-1}: 3426-3261,3056,2955,2890$, 1687-1661, $1604(\mathrm{C}=\mathrm{C}) .{ }^{1} \mathrm{H}$ NMR $\left(\mathrm{DMSO}_{6} \mathrm{~d}_{6} 400 \mathrm{MHz}\right) \delta=1.17(\mathrm{t}, 3 \mathrm{H}, J=6.09 \mathrm{~Hz}$, $\left.\mathrm{OCH}_{2} \underline{\mathrm{CH}}_{3}\right), 4.18\left(\mathrm{q}, 2 \mathrm{H}, J=6.09 \mathrm{~Hz}, \mathrm{OCH}_{2} \mathrm{CH}_{3}\right), 5.90\left(\mathrm{~s}, 2 \mathrm{H}, \mathrm{D}_{2} \mathrm{O}\right.$ exchangeable, $\left.\mathrm{NH}_{2}\right), 7.31-$ $7.99\left(\mathrm{~m}, 10 \mathrm{H}, 2 \mathrm{C}_{6} \mathrm{H}_{5}\right), 10.01\left(\mathrm{~s}, 1 \mathrm{H}, \mathrm{D}_{2} \mathrm{O}\right.$ exchangeable, $\left.\mathrm{NH}\right) .{ }^{13} \mathrm{C}$ NMR (DMSO-d $\left.675 \mathrm{MHz}\right) \delta$ : $16.5\left(\mathrm{OCH}_{2} \mathrm{CH}_{3}\right), 50.2\left(\mathrm{OCH}_{2} \mathrm{CH}_{3}\right), 119.4,119.9,121.9,123.8,124.6,125.1,125.5,126.2$, 132.3, 133.1, 134.6, 135.3 (two $\mathrm{C}_{6} \mathrm{H}_{5}$, thiophene C), 165.8, $167.1(2 \mathrm{C}=\mathrm{O})$. EIMS m/z $366[\mathrm{M}]^{+}$ (28). Anal. calcd. for $\mathrm{C}_{20} \mathrm{H}_{18} \mathrm{~N}_{2} \mathrm{O}_{3} \mathrm{~S}$ (366.43): C, 65.55; H, 4.95; N, 7.64; S, 8.75. Found: C, 65.72; H, 4.73; N, 7.73; S, 8.93.

General procedure for the synthesis of the 4-(phenylcarbamoyl) thiophene derivatives 16a,b

To a solution of compound 2 (2.39 g, $0.01 \mathrm{~mol})$ in dimethylformamide $(30 \mathrm{~mL})$ containing potassium hydroxide $(0.56 \mathrm{~g}, 0.01 \mathrm{~mol})$ phenylisothiocyanate was added. The reaction mixture was stirred at room temperature for $24 \mathrm{~h}$ then to the reaction mixture either ethyl chloroacetate $(1.22 \mathrm{~g}, 0.01 \mathrm{~mol})$ or $(0.92 \mathrm{~g}, 0.01 \mathrm{~mol})$ was added. The reaction mixture was stirred at room temperature for an additional $24 \mathrm{~h}$ then poured onto ice/water containing few drops of hydrochloric acid (till $\mathrm{pH} 6$ ) and the formed solid product was collected by filtration.

Ethyl 3-phenyl-5-(phenylamino)-4-(phenylcarbamoyl)thiophene-2-carboxylate (16a). Yellow crystals (1,4-dioxane), yield 86\% (3.80 g), mp 160-163 ${ }^{\circ} \mathrm{C}$. IR $v_{\max } \mathrm{cm}^{-1}$ : 3397-3229, 3048, 2986, 2890, 1688-1668, 1624. ${ }^{1} \mathrm{H}$ NMR (DMSO-d $\left.6400 \mathrm{MHz}\right) \delta=1.21$ (t, 3H, $J=7.21 \mathrm{~Hz}$, $\left.\mathrm{OCH}_{2} \mathrm{CH}_{3}\right), 3.87\left(\mathrm{q}, 2 \mathrm{H}, \mathrm{J}=7.21 \mathrm{~Hz}, \underline{\mathrm{OCH}_{2}} \mathrm{CH}_{3}\right), 7.43-7.49\left(\mathrm{~m}, 15 \mathrm{H}, 3 \mathrm{C}_{6} \mathrm{H}_{5}\right), 8.20,10.39(2 \mathrm{~s}$, $2 \mathrm{H}, \mathrm{D}_{2} \mathrm{O}$ exchangeable, 2NH). ${ }^{13} \mathrm{C} \mathrm{NMR}\left(\mathrm{DMSO}-\mathrm{d}_{6} 75 \mathrm{MHz}\right) \delta=16.6\left(\mathrm{OCH}_{2} \mathrm{CH}_{3}\right), 50.6$ $\left(\mathrm{OCH}_{2} \mathrm{CH}_{3}\right), 120.0,121.8,122.6,123.8,123.9,124.2,124.5,125.2,125.3,125.8,126.3,126.9$, 130.4, 133.1, 138.0, 139.2 (three $\mathrm{C}_{6} \mathrm{H}_{5}$ and thiophene C), 165.8, 167.1 (2C=O). EIMS m/z 366 $[\mathrm{M}]^{+}$(28). Anal. calcd. for $\mathrm{C}_{26} \mathrm{H}_{22} \mathrm{~N}_{2} \mathrm{O}_{3} \mathrm{~S}$ (442.53): C, 70.57; H, 5.01; N, 6.33; S, 7.24. Found: C, 70.29; H, 4.87; N, 6.58; S, 7.42.

5-Methyl-N,4-diphenyl-2-(phenylamino)thiophene-3-carboxamide (16b). Orange crystals (1,4dioxane), yield $79 \%$ (3.25 g), $\mathrm{mp} 175-177^{\circ} \mathrm{C}$. IR $v_{\max } \mathrm{cm}^{-1}: 3453-3422,3054,2932,1720-1668$. ${ }^{1} \mathrm{H}$ NMR (DMSO-d $\left.\mathrm{d}_{6} 400 \mathrm{MHz}\right): \delta=3.56\left(\mathrm{~s}, 3 \mathrm{H}, \mathrm{CH}_{3}\right), 7.24-7.56\left(\mathrm{~m}, 15 \mathrm{H}, 3 \mathrm{C}_{6} \mathrm{H}_{5}\right), 10.51,11.30$ $\left(2 \mathrm{~s}, 2 \mathrm{H}, \mathrm{D}_{2} \mathrm{O}\right.$ exchangeable, 2NH). ${ }^{13} \mathrm{C}$ NMR (DMSO-d $\left.\mathrm{d}_{6} 75 \mathrm{MHz}\right) \delta: 16.6\left(\mathrm{CH}_{3}\right), 119.2,120.5$, $120.8,121.2,121.7,122.5,123.6,123.8,124.8,125.3,125.6,126.2,130.4,132.5,133.8,134.1$ (three benzene C, thiophene C), 167.8, $172.5(2 \mathrm{C}=\mathrm{O})$. EIMS m/z $412[\mathrm{M}]^{+}(20)$. Anal. calcd. for $\mathrm{C}_{25} \mathrm{H}_{20} \mathrm{~N}_{2} \mathrm{O}_{2} \mathrm{~S}$ (412.51): C, 72.79; H, 4.89; N, 6.79; S, 7.77. Found: C, 72.95; H, 4.63; N, 6.56; S, 8.04 .

\section{2-(1H-Benzo[d] imidazol-2-yl)-1-phenylethanone (18)}

Equimolar amounts of ethyl benzoylacetate $(1.92 \mathrm{~g}, 0.01 \mathrm{~mol})$ and o-phenylenediamine $(1.09 \mathrm{~g}$, $0.01 \mathrm{~mol}$ ) were heated at $120^{\circ} \mathrm{C}$ in the dry conditions in an oil bath for $10 \mathrm{~min}$ then the reaction mixture was left to cool. The formed solid product was triturated with diethylether then 
collected by filtration. Pale yellow crystals (1,4-dioxane), yield $72 \%(1.69 \mathrm{~g}), \mathrm{mp} \quad 180-183{ }^{\circ} \mathrm{C}$. IR $v_{\max } \mathrm{cm}^{-1}: 3437-3302,3053,2974,1680,1615 .{ }^{1} \mathrm{H}$ NMR (DMSO-d $\left.6400 \mathrm{MHz}\right): \delta=3.50(\mathrm{~s}$, $2 \mathrm{H}, \mathrm{CH}_{2}$ ), 7.20-7.55 (m, 9H, $\left.\mathrm{C}_{6} \mathrm{H}_{5}, \mathrm{C}_{6} \mathrm{H}_{4}\right), 10.45$ (s, $1 \mathrm{H}, \mathrm{D}_{2} \mathrm{O}$ exchangeable, $\left.\mathrm{NH}\right) .{ }^{13} \mathrm{C}$ NMR $\left(\right.$ DMSO-d $\left._{6} 75 \mathrm{MHz}\right): \delta=49.8\left(\mathrm{CH}_{2}\right), 118.2,123.8,124.9,125.0,125.3,126.0,126.4,126.8$, 128.3, 129.6 (benzene, indole C), $164.8(\mathrm{C}=\mathrm{O}), 167.2(\mathrm{C}=\mathrm{N})$. EIMS m/z $236[\mathrm{M}]^{+}$(90). Anal. calcd. for $\mathrm{C}_{15} \mathrm{H}_{12} \mathrm{~N}_{2} \mathrm{O}$ (236.27): C, 76.25; H, 5.12; N, 11.86. Found: C, 76.39; H, 4.82; N, 11.66.

General procedure for the synthesis of the benzo[d] imidazol derivatives 20a-c

Either benzaldehyde (1.06 g, $0.01 \mathrm{~mol})$ 4-methoxybanzaldehyde (1.36 g, $0.01 \mathrm{~mol})$ or 4chlorobenzaldehyde $(1.40 \mathrm{~g}, 0.01 \mathrm{~mol})$ was added to a solution of compound $18(2.36 \mathrm{~g}, 0.01$ $\mathrm{mol})$ in 1,4-dioxane $(40 \mathrm{~mL})$ containing piperidine $(0.50 \mathrm{~mL})$. The reaction mixture, in each case, was heated under reflux for $3 \mathrm{~h}$ then poured onto ice/water containing few drops of hydrochloric acid. The formed oily product was triturated with ethanol and the precipitated product was collected by filtration.

2-(1H-Benzo[d] imidazol-2-yl)-1,3-diphenylprop-2-en-1-one (20a). Pale yellow crystals (1,4dioxane), yield $68 \%(2.20 \mathrm{~g}), \mathrm{mp} 183-187{ }^{\circ} \mathrm{C}$. IR $v_{\max } \mathrm{cm}^{-1}: 3537-3425,3053,1680,1615 .{ }^{1} \mathrm{H}$ NMR (DMSO-d $6400 \mathrm{MHz}): \delta=6.06(\mathrm{~s}, 1 \mathrm{H}, \mathrm{CH}), 7.26-7.55\left(\mathrm{~m}, 14 \mathrm{H}, 2 \mathrm{C}_{6} \mathrm{H}_{5}, \mathrm{C}_{6} \mathrm{H}_{4}\right), 10.54(\mathrm{~s}$, $1 \mathrm{H}, \mathrm{D}_{2} \mathrm{O}$ exchangeable $\left.\mathrm{NH}\right) .{ }^{13} \mathrm{C}$ NMR (DMSO-d $\left.675 \mathrm{MHz}\right) \delta: 85.1,112.3(\mathrm{C}=\mathrm{C}), 120.3,122.2$, 122.6, 123.1, 123.5, 124.0, 124.6, 125.0, 125.4, 125.7, 127.5, $127.3\left(2 \mathrm{C}_{6} \mathrm{H}_{5}, \mathrm{C}_{6} \mathrm{H}_{4}\right), 160.2(\mathrm{CO})$, $173.4(\mathrm{C}=\mathrm{N})$. EIMS m/z 324[M] $]^{+}(48)$; anal. calcd. for $\mathrm{C}_{22} \mathrm{H}_{16} \mathrm{~N}_{2} \mathrm{O}$ (324.38): C, 81.46; H, 4.97; N, 8.64. Found: C, 81.66; H, 5.26; N, 8.47.

2-(1H-Benzo[d]imidazol-2-yl)-3-(4-methoxyphenyl)-1-phenylprop-2-en-1-one (20b). Orange crystals (1,4-dioxane), yield 78\% (2.76 g), mp 145-147 ${ }^{\circ} \mathrm{C}$. IR $v_{\max } \mathrm{cm}^{-1}: 3424-3244,3058$, 1710, 1666. ${ }^{1} \mathrm{H}$ NMR (DMSO-d $\left.6400 \mathrm{MHz}\right) \delta=3.50\left(\mathrm{~s}, 3 \mathrm{H}, \mathrm{CH}_{3}\right), 6.08$ (s, 1H, CH), 7.26-7.55 $\left(\mathrm{m}, 13 \mathrm{H}, \mathrm{C}_{6} \mathrm{H}_{5}, 2 \mathrm{C}_{6} \mathrm{H}_{4}\right), 10.54$ (s, $1 \mathrm{H}, \mathrm{D}_{2} \mathrm{O}$ exchangeable $\left.\mathrm{NH}\right) .{ }^{13} \mathrm{C}$ NMR (DMSO-d $\left.675 \mathrm{MHz}\right) \delta$ : $38.7\left(\mathrm{CH}_{3}\right), 89.2,113.8(\mathrm{C}=\mathrm{C}), 120.1,122.4,122.7,123.0,123.3,124.1,124.5,125.2,125.8$, 125.3, 127.1, $127.2\left(\mathrm{C}_{6} \mathrm{H}_{5} .2 \mathrm{C}_{6} \mathrm{H}_{4}\right), 161.2(\mathrm{CO}), 172.8(\mathrm{C}=\mathrm{N})$. EIMS m/z $354[\mathrm{M}]^{+}$(25). Anal. calcd. for $\mathrm{C}_{23} \mathrm{H}_{18} \mathrm{~N}_{2} \mathrm{O}_{2}$ (354.41): C, 77.95; H, 5.12; N, 7.90. Found: C, 78.28; H, 5.08; N, 8.21.

2-(1H-Benzo[d] imidazol-2-yl)-3-(4-chlorophenyl)-1-phenylprop-2-en-1-one (20c). Yellow crystals (1,4-dioxane), yield $72 \%(2.57 \mathrm{~g}), \mathrm{mp} 160-163{ }^{\circ} \mathrm{C}$. IR $v_{\max } \mathrm{cm}^{-1}$ : 3423-3294, 3055, 1720, 1660. ${ }^{1} \mathrm{H}$ NMR (DMSO-d $\left.\mathrm{d}_{6} 400 \mathrm{MHz}\right) \delta=6.08(\mathrm{~s}, 1 \mathrm{H}, \mathrm{CH}), 7.26-7.55\left(\mathrm{~m}, 13 \mathrm{H}, \mathrm{C}_{6} \mathrm{H}_{5}\right.$, $\left.2 \mathrm{C}_{6} \mathrm{H}_{4}\right), 10.54\left(\mathrm{~s}, 1 \mathrm{H}, \mathrm{D}_{2} \mathrm{O}\right.$ exchangeable $\left.\mathrm{NH}\right) .{ }^{13} \mathrm{C}$ NMR (DMSO-d $\left.\mathrm{d}_{6} 75 \mathrm{MHz}\right) \delta=87.3,112.6$ $(\mathrm{C}=\mathrm{C}), 119.2,122.4,122.9,123.0,123.3,124.2,124.8,125.3,125.9,126.1,127.7,128.6\left(\mathrm{C}_{6} \mathrm{H}_{5}\right.$. $\left.2 \mathrm{C}_{6} \mathrm{H}_{4}\right), 161.4(\mathrm{CO}), 171.3(\mathrm{C}=\mathrm{N})$. EIMS m/z $358[\mathrm{M}]^{+}(38)$; anal. calcd. for $\mathrm{C}_{22} \mathrm{H}_{15} \mathrm{ClN}_{2} \mathrm{O}$ (358.83): C, 73.64; H, 4.21; N, 7.81. Found: C, 73.75; H, 4.52; N, 7.95.

\section{2-(3,5-Diphenyl-1H-pyrazol-4-yl)-1H-benzo[d] imidazole (21)}

To a solution of compound 20a $(3.24 \mathrm{~g}, 0.01 \mathrm{~mol})$ in 1,4-dioxane $(40 \mathrm{~mL})$ hydrazine hydrate $(0.50 \mathrm{~g}, 0.01 \mathrm{~mol})$ was added. The reaction mixture was heated under reflux for $2 \mathrm{~h}$ then poured onto ice/water containing few drops of hydrochloric acid and the formed solid product was collected by filtration. Yellow crystals $(\mathrm{EtOH})$, yield $68 \%(2.28 \mathrm{~g}), \mathrm{mp} 221-223{ }^{\circ} \mathrm{C}$. IR $v_{\max }$ $\mathrm{cm}^{-1}: 3440-3302,3054,1662,1608 .{ }^{1} \mathrm{H}$ NMR (DMSO-d $\left.6400 \mathrm{MHz}\right) \delta=7.28-7.74(\mathrm{~m}, 14 \mathrm{H}$, $\left.2 \mathrm{C}_{6} \mathrm{H}_{5}, \mathrm{C}_{6} \mathrm{H}_{4}\right), 10.12,11.20\left(2 \mathrm{~s}, 2 \mathrm{H}, \mathrm{D}_{2} \mathrm{O}\right.$ exchangeable, $\left.2 \mathrm{NH}\right),{ }^{13} \mathrm{C}$ NMR (DMSO-d $\left.675 \mathrm{MHz}\right) \delta$ : $118.3,121.5,121.9,122.6,123.8,124.3,124.5,125.8,126.0,126.2,127.6,130.6,139.2,140.4$ $\left(2 \mathrm{C}_{6} \mathrm{H}_{5}, \mathrm{C}_{6} \mathrm{H}_{4}\right.$, pyrazole C), 163.4, 170.0 (two $\mathrm{C}=\mathrm{N}$ ). EIMS m/z $336[\mathrm{M}]^{+}$(66); anal. calcd. for $\mathrm{C}_{22} \mathrm{H}_{16} \mathrm{~N}_{4}$ (336.40): C, 78.55; H, 4.79; N, 16.66. Found: C, 78.39; H, 4.86; N, 16.41 . 
1-Amino-4-benzoyl-3-(4-methoxyphenyl)benzo[4,5]imidazo[1,2-a]pyridine-2-carbonitrile (22)

Malononitrile $(0.66 \mathrm{~g}, 0.01 \mathrm{~mol})$ was added to a solution of compound $20 \mathrm{~b}(3.54 \mathrm{~g}, 0.01 \mathrm{~mol})$ in 1,4-dioxane $(40 \mathrm{~mL})$ containing triethylamine $(0.50 \mathrm{~mL})$. The reaction mixture was heated under reflux for $4 \mathrm{~h}$ then evaporated under vacuum. The remaining product was triturated with ethanol and the formed solid product was collected by filtration. Yellow crystals (EtOH), yield $73 \%$ (3.05 g), mp 182-185 ${ }^{\circ} \mathrm{C}$. IR $v_{\max } \mathrm{cm}^{-1}: 3438-3304,3054,2200,1687,1662,1614 .{ }^{1} \mathrm{H}$ NMR $\left(\mathrm{DMSO}_{6} \mathrm{~d}_{6} 400 \mathrm{MHz}\right) \delta=3.50\left(\mathrm{~s}, 3 \mathrm{H}, \mathrm{OCH}_{3}\right), 5.02\left(\mathrm{~s}, 2 \mathrm{H}, \mathrm{D}_{2} \mathrm{O}\right.$ exchangeable, $\left.\mathrm{NH}_{2}\right), 7.21-7.55$ $\left(\mathrm{m}, 13 \mathrm{H}, \mathrm{C}_{6} \mathrm{H}_{5}, 2 \mathrm{C}_{6} \mathrm{H}_{4}\right),{ }^{13} \mathrm{C} \mathrm{NMR}$ (DMSO-d $\left.675 \mathrm{MHz}\right) \delta: 46.8\left(\mathrm{OCH}_{3}\right), 116.9(\mathrm{CN}), 120.3$, 120.6, 121.5, 121.8, 122.3, 123.2, 123.7, 124.7, 124.8, 125.0, 125.5, 126.7, 130.4, 131.6, 137.7, $139.9\left(\mathrm{C}_{6} \mathrm{H}_{5}, 2 \mathrm{C}_{6} \mathrm{H}_{4}\right.$, pyridine C), $163.2(\mathrm{C}=\mathrm{O}), 166.8(\mathrm{C}=\mathrm{N})$. EIMS m/z $418[\mathrm{M}]^{+}(48)$; anal. calcd. for $\mathrm{C}_{26} \mathrm{H}_{18} \mathrm{~N}_{4} \mathrm{O}_{2}$ (418.54): C, 74.63; H, 4.34; N, 13.39. Found: C, 74.49; H, 4.62; N, 13.61 .

General procedure for the synthesis of the arylhydrazone derivatives $\mathbf{2 4 a} \mathbf{b}$

To a cold solution $\left(0-5{ }^{\circ} \mathrm{C}\right)$ of compound $18(2.36 \mathrm{~g}, 0.01 \mathrm{~mol})$ in ethanol $(50 \mathrm{~mL})$ containing sodium acetate $(3.50 \mathrm{~g}, 0.50 \mathrm{~mol})$ either benzenediazonium chloride $(0.01 \mathrm{~mol})$ or 4-chlorobenzenediazonium chloride ( $0.01 \mathrm{~mol}$ [prepared by adding a cold solution of sodium nitrite $(0.70 \mathrm{~g}$, in water $(10 \mathrm{~mL}))$ to a cold solution $\left(0-5^{\circ} \mathrm{C}\right)$ of either aniline $(0.93 \mathrm{~g}, 0.01 \mathrm{~mol})$ or 4 chloroaniline $(1.27 \mathrm{~g}, 0.01 \mathrm{~mol})$ in concentrated hydrochloric acid $(12 \mathrm{~mL})$ with continuous stirring] was added with continuous stirring. The whole reaction mixture was left at room temperature for $1 \mathrm{~h}$ then, the formed solid product was collected by filtration.

2-(1H-Benzo[d] imidazol-2-yl)-1-phenyl-2-(2-phenylhydrazono)ethanone (24a). Orange crystals (EtOH), yield 88\% (2.99 g), mp 167-169 ${ }^{\circ} \mathrm{C}$. IR $v_{\max } \mathrm{cm}^{-1}: 3440-3299,3053,1668,1610 .{ }^{1} \mathrm{H}$ NMR (DMSO-d $6400 \mathrm{MHz}) \delta=7.26-7.55\left(\mathrm{~m}, 14 \mathrm{H}, 2 \mathrm{C}_{6} \mathrm{H}_{5}, \mathrm{C}_{6} \mathrm{H}_{4}\right), 8.05,10.55\left(2 \mathrm{~s}, 2 \mathrm{H}, \mathrm{D}_{2} \mathrm{O}\right.$ exchangeable, $2 \mathrm{NH}$ ). ${ }^{13} \mathrm{C}$ NMR (DMSO-d 6 $\left.75 \mathrm{MHz}\right) \delta: 121.0,121.4,122.6,123.1,123.5,124.2$, $124.6,125.0,125.3,126.0,128,2,128.4,130.4,130.9,137.7,139.9\left(2 \mathrm{C}_{6} \mathrm{H}_{5}, \mathrm{C}_{6} \mathrm{H}_{4}\right), 163.0$ $(\mathrm{C}=\mathrm{O}), 166.8,168.4(2 \mathrm{C}=\mathrm{N})$. EIMS m/z $340[\mathrm{M}]^{+}(28)$; anal. calcd. for $\mathrm{C}_{21} \mathrm{H}_{16} \mathrm{~N}_{4} \mathrm{O}(340.13)$ : $\mathrm{C}$, 74.10; H, 4.74; N, 16.46. Found: C, 74.33; H, 4.59; N, 16.79.

2-(1H-Benzo[d] imidazol-2-yl)-1-phenyl-2-(2-(4-chlorophenylhydrazono)ethanone

(24b). Orange crystals from $(\mathrm{EtOH})$, yield $80 \%(2.99 \mathrm{~g}), \mathrm{mp} 155-157^{\circ} \mathrm{C}$. IR $v_{\max } \mathrm{cm}^{-1}: 3442-3302$, $3055,1666,1610 .{ }^{1} \mathrm{H}$ NMR (DMSO-d $\left.\mathrm{d}_{6} 400 \mathrm{MHz}\right) \delta=7.26-7.58\left(\mathrm{~m}, 13 \mathrm{H}, \mathrm{C}_{6} \mathrm{H}_{5}, 2 \mathrm{C}_{6} \mathrm{H}_{4}\right), 8.08$, 10.55 (2s, 2H, $\mathrm{D}_{2} \mathrm{O}$ exchangeable, 2NH). ${ }^{13} \mathrm{C}$ NMR (DMSO-d $\left.675 \mathrm{MHz}\right) \delta: 120.4,121.3,122.7$, $123.3,123.8,124.0,124.4,125.1,125.5,126.4,128.1,128.5,130.7,130.4,138.2,139.5\left(\mathrm{C}_{6} \mathrm{H}_{5}\right.$, $\left.2 \mathrm{C}_{6} \mathrm{H}_{4}\right), 163.2(\mathrm{C}=\mathrm{O}), 166.8,168.6(2 \mathrm{C}=\mathrm{N})$. EIMS m/z $374[\mathrm{M}]^{+}(56)$. Anal. calcd. for $\mathrm{C}_{21} \mathrm{H}_{15} \mathrm{~N}_{4} \mathrm{O}$ (374.83): C, 67.29; H, 4.03; N, 14.95. Found: C, 67.51; H, 3.86; N, 15.04.

\section{(1,3-Diaminobenzo[4,5]imidazo[1,2-a]pyridin-4-yl)(phenyl)methanone (25)}

Malononitrile $(0.66 \mathrm{~g}, 0.01 \mathrm{~mol})$ was added to a solution of compound 18 (2.36 g, $0.01 \mathrm{~mol})$ or $3 \mathbf{b}(2.30 \mathrm{~g}, 0.01 \mathrm{~mol})$ in 1,4-dioxane $(40 \mathrm{~mL})$ containing triethylamine $(0.50 \mathrm{~mL})$. The reaction mixture was heated under reflux for $4 \mathrm{~h}$ then poured onto ice/water and the formed solid product was collected by filtration. Yellow crystals (EtOH), yield 75\% $(2.26 \mathrm{~g}), \mathrm{mp} 190-193{ }^{\circ} \mathrm{C}$. IR $v_{\max }$ $\mathrm{cm}^{-1}: 3426-3201,3055,1670,1570 .{ }^{1} \mathrm{H}$ NMR (DMSO-d $\left.6400 \mathrm{MHz}\right) \delta=4.10,5.40\left(2 \mathrm{~s}, 4 \mathrm{H}, \mathrm{D}_{2} \mathrm{O}\right.$ exchangeable, 2NH $)$, 7.26-7.55 $\left(\mathrm{m}, 10 \mathrm{H}, \mathrm{C}_{6} \mathrm{H}_{5}, \mathrm{C}_{6} \mathrm{H}_{4}\right.$, pyridine $\left.\mathrm{H}-3\right) .{ }^{13} \mathrm{C}$ NMR (DMSO- $\mathrm{d}_{6} 75$ MHz) $\delta=116.2,120.3,120.4,121.1,122.3,122.7,123.5,123.9,124.8,128.1,128.6,130.4$, 142,3, 142.6, $152.6\left(\mathrm{C}_{6} \mathrm{H}_{5}, \mathrm{C}_{6} \mathrm{H}_{4}\right.$, pyridine $\left.\mathrm{C}\right), 165.7(\mathrm{C}=\mathrm{O}), 171.3(\mathrm{C}=\mathrm{N})$. EIMS: $\mathrm{m} / \mathrm{z}=302$ $[\mathrm{M}]^{+}$(24); anal. calcd. for $\mathrm{C}_{18} \mathrm{H}_{14} \mathrm{~N}_{4} \mathrm{O}$ (302.34): C, 71.51; H, 4.67; N, 18.53. Found: C, 71.39; H, $4.48 ; \mathrm{N}, 18.71$. 
General procedure for the synthesis of the benzo[4,5] imidazo[1,2-a]pyridine derivatives 26a,b

Either acetylacetone $(1.0 \mathrm{~g}, 0.01 \mathrm{~mol})$ or ethyl acetoacetate $(1.30 \mathrm{~g}, 0.01 \mathrm{~mol})$ to a solution of compound $18(2.36 \mathrm{~g}, 0.01 \mathrm{~mol})$ in 1,4-dioxane $(40 \mathrm{~mL})$ containing triethylamine $(0.50 \mathrm{~mL})$. The reaction mixture was heated under reflux for $2 \mathrm{~h}$ then poured onto ice/water and the formed solid product was collected by filtration.

(1,3-Dimethylbenzo[4,5]imidazo[1,2-a]pyridine-4-yl)(phenyl)methanone (26a). Yellow crystals (EtOH), yield 68\% (2.10 g), mp 130-133 ${ }^{\circ} \mathrm{C} . \mathrm{IR} v_{\max } \mathrm{cm}^{-1}: 3058,1720,1620 .{ }^{1} \mathrm{H}$ NMR (DMSO$\left.\mathrm{d}_{6} 400 \mathrm{MHz}\right) \delta=2.42,3.34\left(2 \mathrm{~s}, 6 \mathrm{H}, 2 \mathrm{CH}_{3}\right), 6.61(\mathrm{~s}, 1 \mathrm{H}$, pyridine $\mathrm{H}-3), 7.27-7.36\left(\mathrm{~m}, 9 \mathrm{H}, \mathrm{C}_{6} \mathrm{H}_{5}\right.$, $\left.\mathrm{C}_{6} \mathrm{H}_{4}\right) .{ }^{13} \mathrm{C}$ NMR (DMSO-d $\left.675 \mathrm{MHz}\right) \delta=24.3,26.2\left(2 \mathrm{CH}_{3}\right), 118.6,121.4,121.8,123.5,124.5$, $125.3,125.8,126.1,127,3,129.2,130.4,140.5,143.0,150.3\left(\mathrm{C}_{6} \mathrm{H}_{5}, \mathrm{C}_{6} \mathrm{H}_{4}\right.$, pyridine C), 165.5 $(\mathrm{C}=\mathrm{O}), 167.2(\mathrm{C}=\mathrm{N})$. EIMS m/z $300[\mathrm{M}]^{+}$(68). Anal. calcd. for $\mathrm{C}_{21} \mathrm{H}_{16} \mathrm{~N}_{4} \mathrm{O}$ (300.36): C, 79.98; H, 5.37; N, 9.33. Found: C, 79.69; H, 5.48; N, 9.59.

1-Hydroxy-3-methylbenzo[4,5] imidazo[1,2-a]pyridine-4-yl)(phenyl)methanone (26b). Orange crystals (EtOH), yield $77 \%(2.32 \mathrm{~g}), \mathrm{mp} 145-147{ }^{\circ} \mathrm{C}$. IR $v_{\max } \mathrm{cm}^{-1}: 3435-3125,3058,1696$, 1474. ${ }^{1} \mathrm{H}$ NMR (DMSO-d $\left.\mathrm{D}_{6} 400 \mathrm{MHz}\right) \delta=3.34\left(\mathrm{~s}, 3 \mathrm{H}, \mathrm{CH}_{3}\right), 6.63$ (s, $1 \mathrm{H}$, pyridine $\mathrm{H}-3$ ), 7.27$7.38\left(\mathrm{~m}, 9 \mathrm{H}, \mathrm{C}_{6} \mathrm{H}_{5}, \mathrm{C}_{6} \mathrm{H}_{4}\right), 10.50\left(\mathrm{~s}, 1 \mathrm{H}, \mathrm{D}_{2} \mathrm{O}\right.$ exchangeable, OH). ${ }^{13} \mathrm{C}$ NMR (DMSO-d 6 $75 \mathrm{MHz}$ ) $\delta=24.1\left(\mathrm{CH}_{3}\right), 119.2,122.0,122.8,123.1,123.5,124.7,125.2,126.3,127.3,129.2,132.6$, 142.1, 143.0, $144.6\left(\mathrm{C}_{6} \mathrm{H}_{5}, \mathrm{C}_{6} \mathrm{H}_{4}\right.$, pyridine $), 166.4(\mathrm{C}=\mathrm{O}), 168.2(\mathrm{C}=\mathrm{N})$. EIMS m/z $302[\mathrm{M}]^{+}$ (28); anal. calcd. for $\mathrm{C}_{19} \mathrm{H}_{14} \mathrm{~N}_{2} \mathrm{O}_{2}$ (302.33): C, 75.48; H, 4.67; N, 9.27. Found: C, 75.21; H, 4.59; N, 9.41 .

\section{Chemicals}

Chemical used through the anticancer evaluations of the synthesised compounds were obtained from Sigma Chemical Co. (Saint Louis, USA).

\section{Cell cultures}

The cancer cell lines namely the human gastric cancer (NUGC and HR), human colon cancer (DLD1), human liver cancer (HA22T and HEPG2), human breast cancer (MCF), nasopharyngeal carcinoma (HONE1) and normal fibroblast cells (WI38) were kindly provided by the National Cancer Institute (NCI, Cairo, Egypt). The cytotoxicity of the newly synthesised compounds against the six cancer cell lines and the normal fibroblast were demonstrated through Table 1.

c-Met enzymatic activity of the most active compounds

Using homogeneous time-resolved fluorescence (HTRF) assay as previously reported the c-Met kinase activities of compounds 6b, 7b, 9, 13a, 13b, 16a, 20b, 20c, 24b, 25 and 26b were evaluated. The $\mathrm{IC}_{50}$ 's were expressed through Table 2 using foretinib as the positive control. The data revealed that the three compounds expressed high enzymatic activity toward c-Met with $\mathrm{IC}_{50}$ 's much higher than that of the reference foretinib. 
Table 1 . Cytotoxicity of the newly synthesized compounds against a variety of cancer cell lines $\left[\mathrm{IC}_{50}{ }^{\mathrm{b}}\right.$ $(\mathrm{nM})]$.

\begin{tabular}{|c|c|c|c|c|c|c|c|}
\hline \multirow{2}{*}{ Compound } & \multicolumn{7}{|c|}{ Cytotoxocity (IC50 in nM) } \\
\hline & NUGC & DLDI & HA22T & HEPG2 & HONE1 & $\mathrm{MCF}$ & WI38 \\
\hline $6 \mathbf{a}$ & 2140 & 3228 & 2314 & 2230 & 3219 & 2240 & na \\
\hline 6b & 66 & 80 & 238 & 232 & 470 & 128 & na \\
\hline $7 \mathbf{a}$ & 2201 & 1488 & 2150 & 2068 & 3188 & 2146 & na \\
\hline $7 \mathbf{b}$ & 380 & 120 & 114 & 1340 & 666 & 1504 & na \\
\hline 9 & 222 & 360 & 1128 & 2134 & 1240 & 1280 & na \\
\hline 11a & 2210 & 2395 & 1163 & 1446 & 1178 & 3430 & na \\
\hline $11 b$ & 1320 & 1348 & 129 & 330 & 2177 & 2166 & na \\
\hline $12 a$ & 3270 & 2870 & 2244 & 1252 & 1049 & 2271 & na \\
\hline $12 b$ & 1282 & 1320 & 1160 & 870 & 1140 & 1160 & na \\
\hline 13a & 860 & 1028 & 759 & 1025 & 1254 & 1220 & na \\
\hline 13b & 750 & 539 & 1220 & 299 & 1186 & 1228 & 380 \\
\hline $16 a$ & 182 & 268 & 560 & 2028 & 1130 & 328 & na \\
\hline $16 b$ & 1135 & 1062 & 1011 & 1210 & 466 & 210 & na \\
\hline 18 & 2220 & 2440 & 1128 & 2432 & 2143 & 2056 & na \\
\hline $20 a$ & 1240 & 1562 & 1063 & 1260 & 1619 & 1655 & na \\
\hline $20 b$ & 480 & 682 & 1158 & 1324 & 1460 & 1603 & na \\
\hline $20 c$ & 330 & 268 & 98 & 64 & 1209 & 332 & na \\
\hline 21 & 2262 & 2152 & 1381 & 1060 & 1313 & 1145 & na \\
\hline 22 & 1080 & 150 & 840 & 324 & 360 & 1246 & 665 \\
\hline $24 a$ & 1149 & 2160 & 3261 & 2136 & 2484 & 2868 & na \\
\hline $24 b$ & 380 & 122 & 33 & 669 & 1240 & 890 & na \\
\hline 25 & 487 & 350 & 3340 & 229 & 82 & 262 & na \\
\hline $26 a$ & 1445 & 3110 & 3013 & 3076 & 670 & 2742 & na \\
\hline $26 b$ & 32 & 48 & 260 & 82 & 71 & 559 & na \\
\hline CHS 828 & 25 & 2315 & 2067 & 1245 & 15 & 18 & na \\
\hline
\end{tabular}

${ }^{a}$ NUGC, gastric cancer, DLDI, colon cancer, HA22T, liver cancer, HEPG2, liver cancer; HONEI, nasopharyngeal carcinoma; HR, gastric cancer; MCF, breast cancer; WI38, normal fibroblast cells.

Table 2. c-Met enzymatic activity of the most active compounds.

\begin{tabular}{|c|c|}
\hline Compound No. & $\mathrm{IC}_{50}(\mathrm{nM}) \mathrm{c}-\mathrm{Met}$ \\
\hline $\mathbf{6 b}$ & $0.24 \pm 0.05$ \\
\hline $\mathbf{7 b}$ & $0.36 \pm 0.28$ \\
\hline $\mathbf{9}$ & $0.42 \pm 0.16$ \\
\hline $\mathbf{1 3 a}$ & $0.58 \pm 0.28$ \\
\hline $\mathbf{1 3 b}$ & $0.61 \pm 0.31$ \\
\hline $\mathbf{1 6 a}$ & $0.48 \pm 0.19$ \\
\hline $\mathbf{2 0 b}$ & $0.53 \pm 0.20$ \\
\hline $\mathbf{2 0 c}$ & $0.86 \pm 0.45$ \\
\hline $\mathbf{2 4 b}$ & $0.50 \pm 0.28$ \\
\hline $\mathbf{2 5}$ & $0.73 \pm 0.25$ \\
\hline $\mathbf{2 6}$ & $0.80 \pm 0.32$ \\
\hline & Foretinib1.16 \\
\hline & \\
\hline
\end{tabular}

\section{RESULTS AN DISCUSSION}

The present investigation emphasized mainly on two important things, of these one is to the synthesis of molecules having nitrogen and or sulfur heterocyclic and the other is to determine 


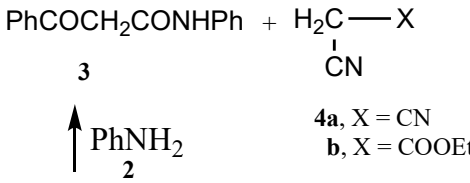
b, $\mathrm{X}=\mathrm{COOE}$

1

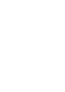

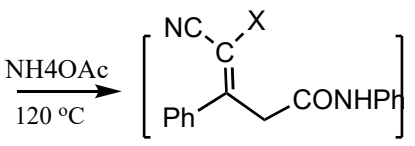

5a, $\mathrm{X}=\mathrm{CN}$ b, $\mathrm{X}=\mathrm{COOE} t$

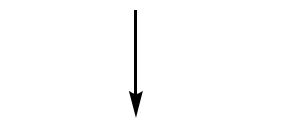<smiles>[X]C1=C(c2ccccc2)CC(=O)N(c2ccccc2)C1=N</smiles>

6a, $\mathrm{X}=\mathrm{CN}$

b, $\mathrm{X}=\mathrm{COOE}$<smiles></smiles><smiles>N#Cc1c(N)nc2c(c(-c3ccccc3)cc(=O)n2-c2ccccc2)c1N</smiles>

9

Scheme 1. Synthesis of compounds $\mathbf{6 a}, \mathbf{b} ; \mathbf{7 a}, \mathbf{b}$ and $\mathbf{9 .}$

their cytotoxicity against cancer and normal cell lines. The synthetic strategies adopted for the synthesis of the intermediates and target compounds are depicted in Schemes 1-4. Nitrogen containing heterocyclic organic compounds having extra keto group show interesting chemical properties as well as biological activity [33]. The reaction of ethyl benzoylacetate (1) with aniline (2) gave the 3-oxo-N,3-diphenylpropanamide (3). Compound 3 reacted with either malononitrile (4a) or ethyl cyanoacetate (4b) to give the 1,2-dihydropyridinederivatives $6 \mathbf{a}$ and $\mathbf{6 b}$, respectively, through the acyclic intermediates $\mathbf{5 a}, \mathbf{b}$. The structure of the latter products was based on analytical and spectral data. Thus, the ${ }^{1} \mathrm{H}$ NMR spectrum of $\mathbf{6 a}$ showed the presence of a singlet at $\delta 4.15 \mathrm{ppm}$ indicating the presence of the $\mathrm{CH}_{2}$ group, a multiplet at $\delta 7.30-7.99 \mathrm{ppm}$ 
for the two phenyl groups and a singlet at $\delta 10.21 \mathrm{ppm}\left(\mathrm{D}_{2} \mathrm{O}\right.$ exchangeable $)$ corresponding to the $\mathrm{NH}$ group. Moreover, the ${ }^{13} \mathrm{C}$ NMR spectrum showed signal at $\delta 49.5$ corresponding to the pyridine $\mathrm{C}-3$, two signals at $\delta 103.6,104.5$ for the $\mathrm{C}=\mathrm{C}$ group, a signal at $\delta 119.5$ for the $\mathrm{CN}$ group, eight signals at $\delta 120.19,132.9,125.8,129.4,129.5,134.5,136,8,139.5$ equivalent to two benzene and pyridine $\mathrm{C}$ and two signals at $\delta 165.9,195.2$ confirming the $\mathrm{C}=\mathrm{O}$ and $\mathrm{C}=\mathrm{N}$ groups. On the other hand, the reaction of compound 3 with either malononitrile or ethyl cyanoacetate in sodium ethoxide solution gave the 1,6-dihydropyridine derivatives $7 \mathbf{a}$ and $7 \mathbf{b}$, respectively. The reaction of compound $\mathbf{3}$ with the 2-aminoprop-1-ene-1,1,3-tricarbonitrile $\mathbf{8}$ gave the 7,8-dihydro-1,8-naphthyridine derivative 9 (Scheme 1) its structure was confirmed on the basis of analytical and spectral data.

The high yield of compound $\mathbf{7 b}$ encouraged us to make further work in order to produce pharmaceutically active fused pyridine derivatives. Thus, the reaction of compound $7 \mathbf{b}$ with either acetylacetone (10a) or ethyl acetoacetate (10b) gave the pyrido[2,3-c]pyridine derivatives 11a and $11 b$, respectively. Furthermore, compound $7 \mathbf{b}$ reacted with either malononitrile or ethyl cyanoacetate to give the pyrido[2,3-c]pyridine derivatives $\mathbf{1 2 a}$ and $\mathbf{1 2 b}$, respectively. The analytical and spectral data of 11a,b and 12a,b were in agreement with their proposed structures.<smiles>NC1=C(C(=O)c2ccccc2)C(=O)N(c2ccccc2)C(=O)C1</smiles>

$7 \mathbf{b}$

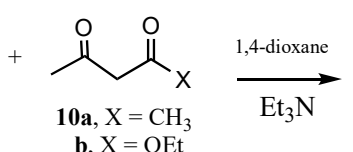

b, $\mathrm{X}=\mathrm{OEt}$<smiles>[Y]c1cc(C)[nH]c2c(C(=O)c3ccccc3)c(=O)n(-c3ccccc3)c(=O)c1-2</smiles>

11a, $\mathrm{Y}=\mathrm{CH}_{3}$

b, $\mathrm{Y}=\mathrm{OH}$<smiles>[R]C1=CC(N)=NC2=C(C(=O)c3ccccc3)C(=O)N(c3ccccc3)C(=O)C12</smiles>

12a, $\mathrm{R}=\mathrm{NH}_{2}$ $\mathrm{b}, \mathrm{R}=\mathrm{OH}$

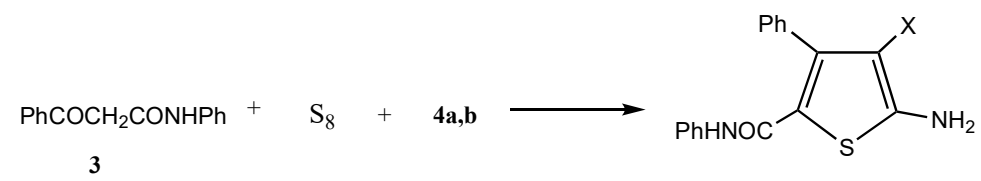

13a, $\mathrm{X}=\mathrm{CN}$

b, $\mathrm{X}=\mathrm{COOEt}$

Scheme 2. Synthesis of compounds 11a,b; 12a,b and 13a,b. 

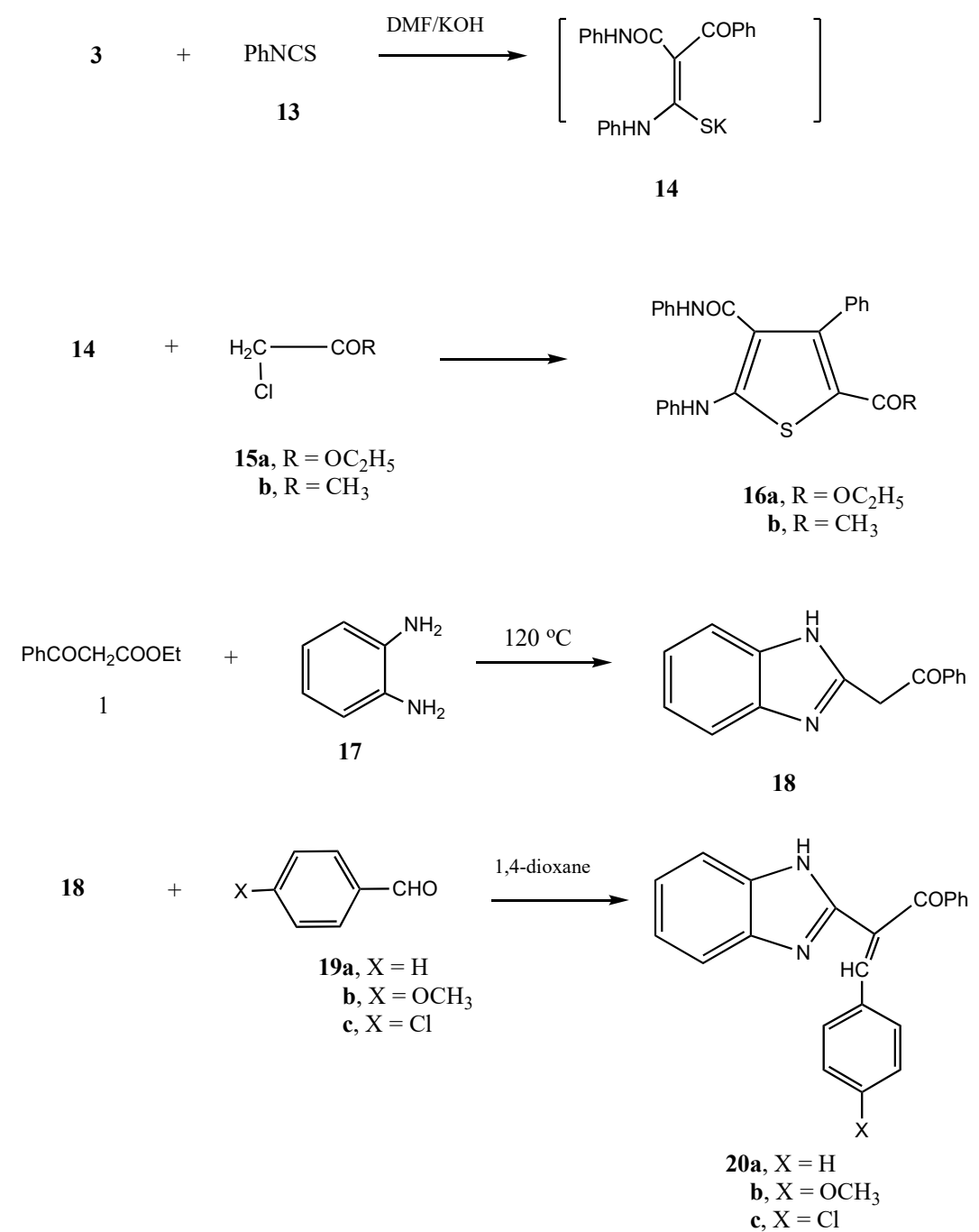

Scheme 3. Synthesis of compounds 16a,b; 19 and 20a-c.

The benzoylbenzanilide was studied to synthesis thiophene through the well known Gewald's thiophene synthesis $[34,35]$. Thus, the reaction of compound $\mathbf{3}$ with elemental sulfur and either malononitrile (4a) and ethyl cyanoacetate (4b) gave the thiophene derivatives 13a and $\mathbf{1 3 b}$, respectively (Scheme 2). On the other hand, compound $\mathbf{3}$ reacted with phenyl isothiocyanate in basic dimethylformamide gave the intermediate potassium sulphide salt $\mathbf{1 4}$. The latter intermediate reacted with either ethyl chloroacetate (15a) or chloroacetone $(\mathbf{1 5 b})$ to give the thiophene derivatives16a and 16b, respectively. The structures of the latter products were established on the basis of analytical and spectral data. Thus, the ${ }^{1} \mathrm{H}$ NMR spectrum of 16a showed a triplet at $\delta 0.71 \mathrm{ppm}$ indicating the presence of the ester $\mathrm{CH}_{3}$ group, a quartet at $\delta 3.87$ for the ester $\mathrm{CH}_{2}$ group, a mutiplet at $\delta$ 7.43-7.49 equivalent to the three phenyl protons and two singlets at $\delta 8.20$ and 10.39 for the two $\mathrm{NH}$ groups. The ${ }^{13} \mathrm{C}$ NMR spectrum showed the 
presence of a signal at $\delta 13.6$ for the $\mathrm{CH}_{3}$ group, a signal at $\delta 39.6$ equivalent to the $\mathrm{CH}_{2}$ group and eleven signals at $\delta 110.0,121.8,125.8,126.3,128.4,129.1,130.4,133.1,138.0,139.2$, $140.2,148.7$ indicating the two $\mathrm{C}_{6} \mathrm{H}_{5}$ and thiophene $\mathrm{C}$ and two signals at $\delta 165.8,195.1$ for the two $\mathrm{C}=\mathrm{O}$ groups.

Next we moved towards the uses of ethyl benzoylacetate to synthesis of benzimidazole derivatives. Thus, compound 1 reacted with orthophenylene diamine in an oil bath $120{ }^{\circ} \mathrm{C}$ to give the 2-(1H-benzo[ $d]$ imidazol-2-yl)-1-phenylethanone (18). The analytical and spectral data of compound 18 were in agreement with its proposed structure. Compound 18 reacted with either benzaldehyde (19a), 4-methoxybenzaldehyde (19b) or 4-chlorobenzaldehyde (19c) to give the benzalidene derivatives $\mathbf{2 0 a - c}$, respectively (Scheme 3 ).

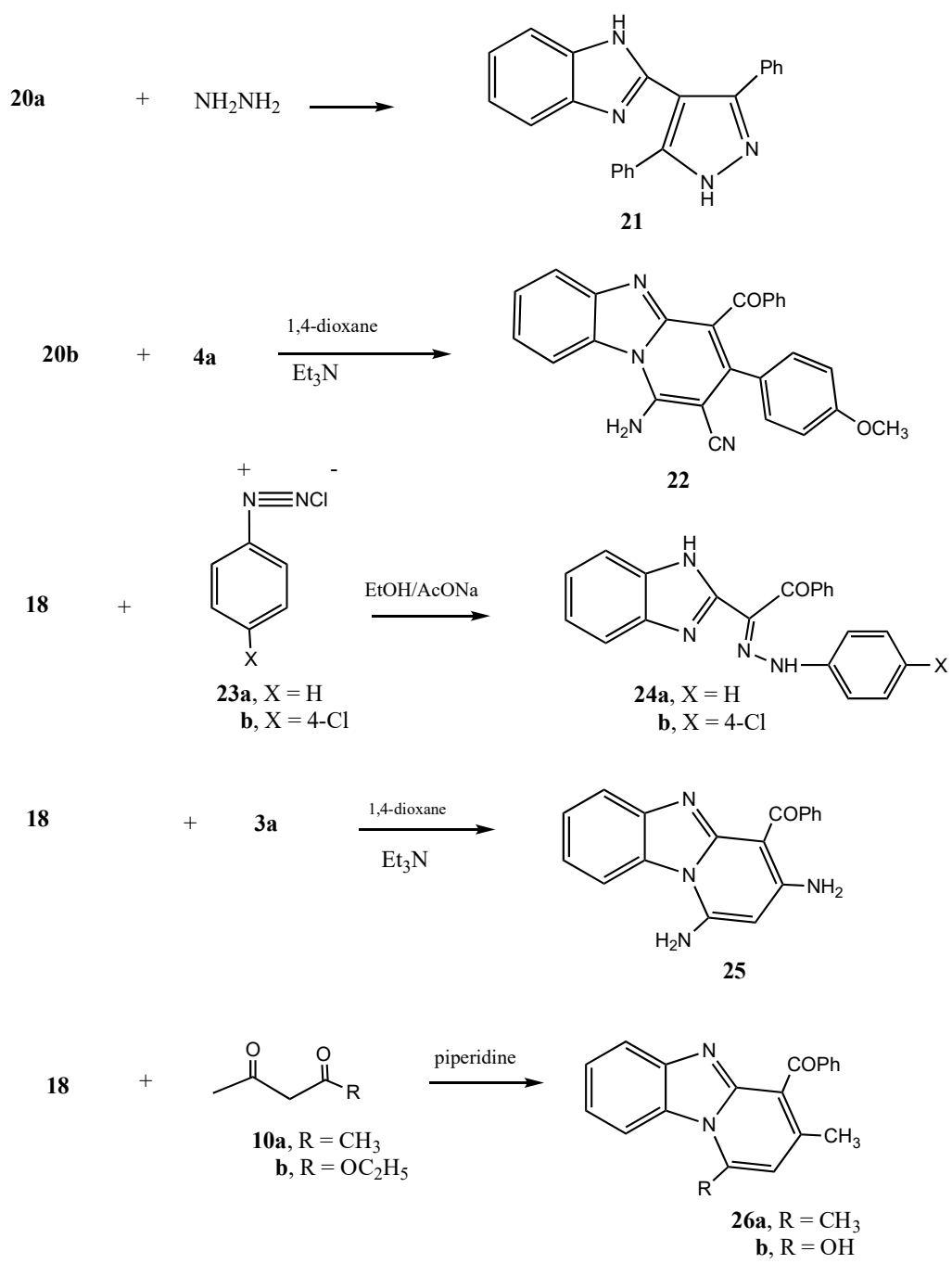

Scheme 4. Synthesis of compounds $21 ; \mathbf{2 4 a}, \mathbf{b} ; \mathbf{2 5}$ and $\mathbf{2 6 a}, \mathbf{b}$.

Bull. Chem. Soc. Ethiop. 2020, 34(2) 
Compound 20a reacted with hydrazine hydrate to give the pyrazole derivative 21 . On the other hand, 20b reacted with malononitrile (3a) to give the 1-amino-4-benzoyl-3-(4methoxyphenyl)benzo[4,5]imidazo[1,2-a]pyridine-2-carbonitrile (22).

Compound 18 reacted with either benzenediazonium chloride (23a) or 4chlorobenzenediazonium chloride (23b) to give the phenylhydrazo derivative $\mathbf{2 4 a}$ and $\mathbf{2 4 b}$, respectively. On the other hand, it reacted with malononitrile (3a) in 1,4-dioxane containing triethylamine to give the benzo[4,5]imidazo[1,2-a]pyridine derivative 25. Similarly compound 18 reacted with either either acetylacetone (10a) or ethyl acetoacetate (10b) to give the benzo[4,5]imidazo[1,2-a]pyridine derivatives $\mathbf{2 6 a}$ and $\mathbf{2 6 b}$, respectively (Scheme 4).

\section{Structure activity relationship}

From Table 1 the newly synthesized compounds were tested against the six cancer cell lines the human gastric cancer (NUGC), human colon cancer (DLD1), human liver cancer (HA22T and HEPG2), human breast cancer (MCF), nasopharyngeal carcinoma (HONE1) and a normal fibroblast cells (WI38). Compounds 6b, 7b, 9, 13a, 13b, 16a, 20b, 20c, 24b, 25 and 26b exhibited optimal cytotoxic effect against cancer cell lines, with $\mathrm{IC}_{50}$ 's in the $\mathrm{nM}$ range. Comparing the cytotoxicity of the 1,2-dihydropyridine derivatives $\mathbf{6 a}$ and $\mathbf{6 b}$, it is obvious that the ethyl ester group present in $\mathbf{6 b}$ is responsible for its high cytotoxicity. On the other hand considering the 1,6-dihydropyridine $7 \mathbf{a}$ and $\mathbf{7 b}$; the latter compound with the $\mathrm{OH}$ group showed high cytotoxicity against the NUGC, DLDI, HA22T and HONE1 cell lines. The 2-(amino(6hydroxy-2-imino-1,4-diphenyl-1,2-dihydropyridin-3-yl)methylene)-malononitrile (9) showed high cytotoxicity against NUGC, DLDI and HEPG2 cell lines. Such high potency of compound 9 is attributed to the presence of the dicyanethylidene moiety. Comparing the cytotoxicity of the 1,6-naphthridine derivatives 11a and 11b, it is obvious that the presence of the $\mathrm{OH}$ group in compound 11b is responsible for its high cytotoxicity against HA22T and HEPG2 cell lines. Such finding was confirmed through the comparison of the cytotoxicity of compounds 12a and 12b, where the last one showed higher cytotoxicity towards the six cancer cell lines. The significant cytotoxicity of $\mathbf{1 2 b}$ appeared against the HEPG2 cell line with $870 \mathrm{nM}$.

For the thiophene derivatives $\mathbf{1 3 a}, \mathbf{b}$ and $\mathbf{1 6 a}, \mathbf{b}$, it is clear from Table 1 that such compounds showed significant cytotoxicity against the cancer cell lines. Compounds $\mathbf{1 3 b}$ and $\mathbf{1 6 a}$ with the special optimal cytotoxicity which is attributed to the presence of the oxygen rich COOEt and $\mathrm{OCH}_{3}$ moeties in both compound, respectively. It is of great interest to compare the cytotoxicity of the un-substituted benzimidazole derivative $\mathbf{1 8}$ and the benzylidene derivatives 20a-c. It is obvious that compound $\mathbf{1 8}$ showed lower cytotoxicity relative to 20a-c. In addition going through the latter compounds, one can notice that the presence of the electronegative groups $\mathrm{OCH}_{3}$ and $\mathrm{Cl}$ in $20 \mathbf{b}$ and 20c, respectively are responsible for the high cytotoxicity of such compounds. Moreover, the $\mathrm{Cl}$ group showed more potency than the $\mathrm{OCH}_{3}$ group as cleared from the greater cytotoxicity of 20c over 20b. The annulated compound 22 showed higher cytotoxicity than the pyrazole derivative 21 . The reaction of the benzimidazole derivative $\mathbf{1 8}$ with arylidenediazonium salts $\mathbf{2 3} \mathbf{a}$ and $\mathbf{2 3} \mathbf{b}$ gave the arylhydrazone derivatives $\mathbf{2 4 a}$ and 24b leading to a remarkable increase of cytotoxicity of $\mathbf{2 4 a}, \mathbf{b}$. In addition compound $\mathbf{2 4 \mathbf { b }}$ with $\mathrm{Cl}$ group showed an optimal cytotoxicity against NUGC, DLDI, HA22T, HEPG2 and MCF cell lines with $\mathrm{IC}_{50}$ 's $380,122,33,669$ and $890 \mathrm{nM}$, respectively. It is of great value to note that compound 24b with the $\mathrm{Cl}$ group showed more potency than 24a. Considering the imidazo[1,2a]pyridine derivatives $\mathbf{2 5}$ and $\mathbf{2 6 a}, \mathbf{b}$ compound $\mathbf{2 6 b}$ showed the highest cytotoxticity among the three compounds. Moreover, the nitrogen rich compound 25 showed higher cytotoxicity than 26a. It is of good worthy to notice that the ethyl 6-hydroxy-2-imino-1,4-diphenyl-1,2dihydropyridine-3-carboxylate $(\mathbf{6 b})$ and the (1-hydroxy-3-methylbenzo[4,5]imidazo[1,2a]pyridine-4-yl)(phenyl)methanone (26b) showed the maximum inhibitory effect towards the six cancer cell lines among the tested compounds. 
It is very clear from our present finding that the heterocyclic systems with halogen substituted pattern $\mathrm{OCH}_{3}, \mathrm{Cl}$ or COOEt show greater cytotoxic property. In every case it was observed that molecules with electronegative substitutions as compounds $\mathbf{6 b}, \mathbf{7 b}, \mathbf{1 3 a}, \mathbf{b} ; \mathbf{1 6} \mathbf{6}, \mathbf{b}$; 20b, 20c, 25, and 26b showed higher cytotoxicity because they were bearing either oxygen or chlorine substituted as well as comprised with similar structural features.

\section{Toxicity}

It is well known that bioactive compounds are often toxic to shrimp larvae. Thus, in order to monitor these chemicals' in vivo lethality to shrimp larvae (Artemia salina), results are given in Table 3 for the compounds which exhibited optimal cytotoxic effect against cancer cell lines which are the ten compounds $6 \mathbf{b}, 7 \mathbf{b}, 13 \mathbf{a}, 13 \mathbf{b}, 16 \mathbf{a}, 16 \mathbf{b}, 20 \mathbf{b}, 20 \mathbf{c}, 25$ and 26b. The shrimp lethality assay is considered as a useful tool for preliminary assessment of toxicity, and it has been used for the detection of fungal toxins, plant extract toxicity, heavy metals, cyanobacteria toxins, pesticides, and cytotoxicity testing of dental materials, natural and synthetic organic compounds [36].

Table 3. Toxicity of the most cytotoxic compounds.

\begin{tabular}{|c|c|c|c|c|c|c|}
\hline $\begin{array}{c}\text { Compound } \\
\text { No. }\end{array}$ & $\begin{array}{c}\text { Cons. } \\
(\mu \mathrm{g} / \mathrm{mL})\end{array}$ & Mortality $^{\mathrm{a}}$ & Toxicity & $\mathrm{LC}_{50}$ & $\begin{array}{c}\text { Upper } \\
95 \% \text { limit }\end{array}$ & $\begin{array}{c}\text { Lower } \\
95 \% \text { limit }\end{array}$ \\
\hline $6 b$ & $\begin{array}{c}10 \\
100 \\
1000\end{array}$ & $\begin{array}{l}0 \\
0 \\
8\end{array}$ & Non toxic & 890.22 & - & - \\
\hline $7 b$ & $\begin{array}{c}10 \\
100 \\
1000\end{array}$ & $\begin{array}{l}2 \\
6 \\
8\end{array}$ & Harmful & 177.63 & 673.42 & 285.41 \\
\hline $13 a$ & $\begin{array}{c}10 \\
100 \\
1000\end{array}$ & $\begin{array}{c}0 \\
5 \\
10 \\
\end{array}$ & Harmful & 213.29 & 66.12 & 22.45 \\
\hline $13 b$ & $\begin{array}{c}10 \\
100 \\
1000\end{array}$ & $\begin{array}{c}1 \\
4 \\
10\end{array}$ & Harmful & 237.39 & 230.62 & 66.80 \\
\hline $16 a$ & $\begin{array}{c}10 \\
100 \\
1000\end{array}$ & $\begin{array}{c}1 \\
6 \\
10\end{array}$ & Harmful & 122.32 & 240.31 & 107.82 \\
\hline $16 \mathrm{~b}$ & $\begin{array}{c}10 \\
100 \\
1000\end{array}$ & $\begin{array}{c}0 \\
1 \\
10\end{array}$ & Harmful & 84.08 & 140.83 & - \\
\hline $20 \mathrm{~b}$ & $\begin{array}{c}10 \\
100 \\
1000\end{array}$ & $\begin{array}{c}0 \\
6 \\
10\end{array}$ & Harmful & 238.40 & 166.23 & 215.80 \\
\hline $20 c$ & $\begin{array}{c}10 \\
100 \\
1000\end{array}$ & $\begin{array}{c}4 \\
8 \\
10\end{array}$ & Very toxic & 12.40 & - & - \\
\hline 25 & $\begin{array}{c}10 \\
100 \\
1000\end{array}$ & $\begin{array}{l}2 \\
5 \\
8\end{array}$ & Very toxic & 24.40 & 365.70 & 213.79 \\
\hline $26 b$ & $\begin{array}{c}10 \\
100 \\
1000\end{array}$ & $\begin{array}{l}0 \\
0 \\
6\end{array}$ & Non toxic & 1000.2 & - & - \\
\hline
\end{tabular}

${ }^{\mathrm{a}}$ Ten organisms (A. salina) tested for each concentration. 
In order to prevent the toxicity results from possible false effects originated from solubility of compounds and DMSO's possible toxicity effect, compounds were prepared by dissolving in DMSO in the suggested DMSO volume ranges. It is clear from Table 3 that compounds $\mathbf{6 b}$ and 26b showed non toxicity against the tested organisms.

\section{Molecular docking}

The molecular studies were carried out using Molecular Operating Environment (MOE 2014). All the minimizations were performed with MOE until a RMSD gradient of $0.01 \mathrm{kcal} / \mathrm{mol} \AA$ with MMFF94X force field and the partial charges were automatically calculated. Docking simulations were performed using the crystal structure of NUGC (PDB ID: 4ASD) which obtained from Protein Data Bank. Enzyme structure was checked for missing atoms, bonds and contacts. Water molecules was removed. Protonate 3D application of MOE was used to add the missing hydrogens and properly assign the ionization states. The ligand molecules were constructed using the builder molecule and were energy minimized. The active site was generated using the MOE-Alpha site finder. Ligands were docked within the active sites using the MOE-Dock The generated poses were energy minimized using the MMFF94x force field. Finally, the optimized poses were ranked using the GBVI/WSA DG free-energy estimates. Docking poses were visually inspected and interactions with binding pocket residues were analyzed.

Docking simulation was carried out to illustrate the binding mode and the interaction of the active compounds $\mathbf{6 b}, \mathbf{7 b}, \mathbf{1 3} \mathbf{a}$ and $\mathbf{1 6} \mathbf{b}$ with the amino acids in the active site of the NUGC. Docking study was performed using the crystal structure of NUGC (PDB ID: 4ASD) [20] which has co-crystallized ligand (sorafenib, BAX) as inhibitor inside its active site. In the beginning, docking study was validated by re-docking of co-crystallized ligand (BAX) inside the active site of NUGC. The re-docking of the co-crystallized ligand, BAX, was carried out to indicate the suitability of the used protocol for the planned docking study. The validation method was achieved by removing the bound ligand from the complex followed by its docking back into the binding site, which yielded root mean square deviation values RMSD of $0.88 \AA$ with energy score (S) $-9.94 \mathrm{kcal} / \mathrm{mol}$. The top pose obtained from the MOE docking simulation showed the interactions of the co-crystallized ligand, BAX, with the key amino acids inside the active site. Docking ligand interaction and electrostatic map of compounds $6 \mathbf{b}, 7 \mathbf{b}, \mathbf{1 3 a}$ and $\mathbf{1 6} \mathbf{b}$ were indicated through Figures 2-5, respectively.

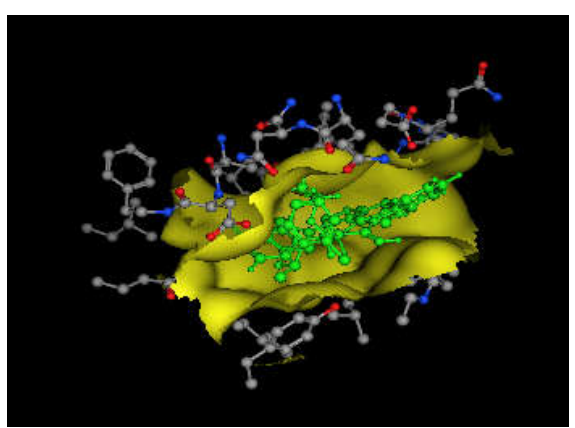

(A)

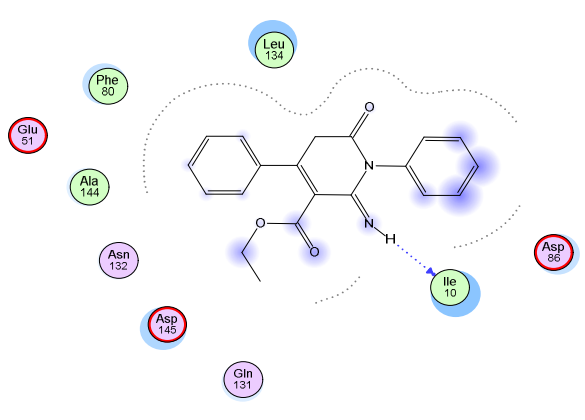

(B) 


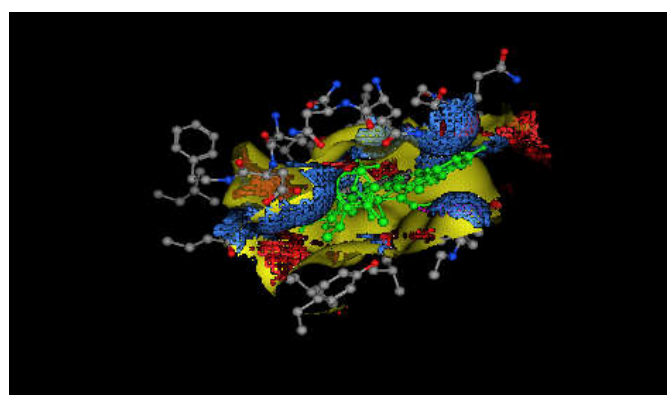

(C)

Figure 2. (A) Docking of compound $\mathbf{6 b}$, (B) Ligand interaction of compound $\mathbf{6 b}$, and (C) electrostatic map of compound $\mathbf{6 b}$. Energy of compound $\mathbf{6 b}=-24.66$.

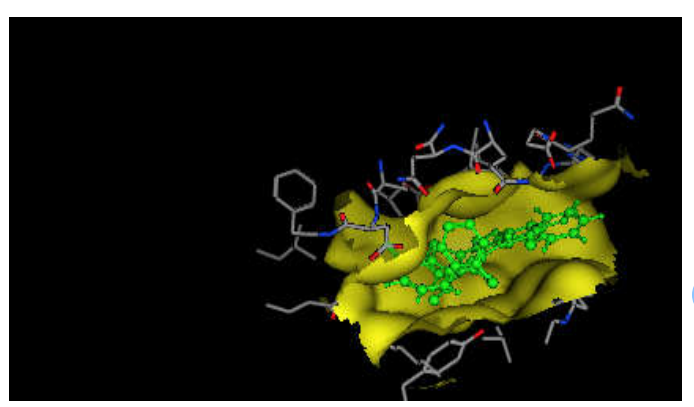

(A)

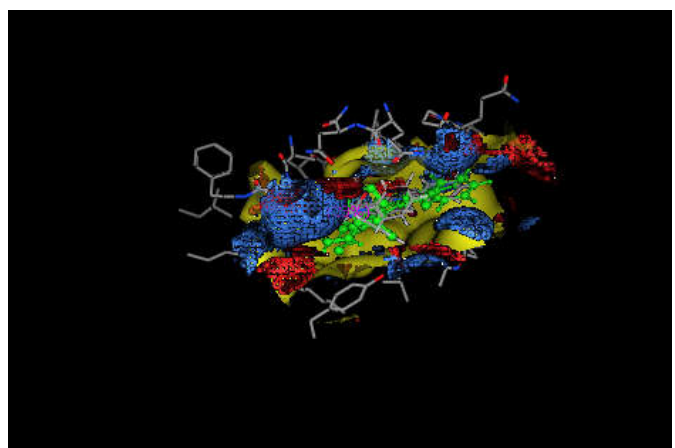

(C)

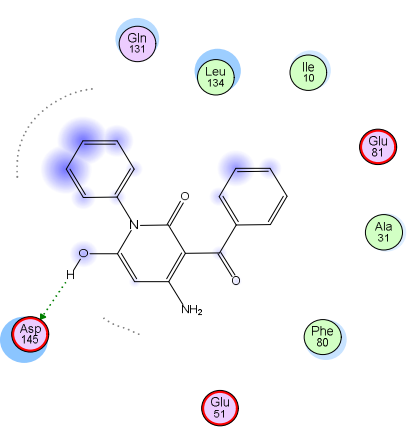

(B)

Figure 3. (A) Docking of compound $7 \mathbf{b}$, (B) ligand interaction of compound $7 \mathbf{b}$, and (C) electrostatic map of $\mathbf{7 b}$. Energy of compound $\mathbf{7 b}=-20.26$. 


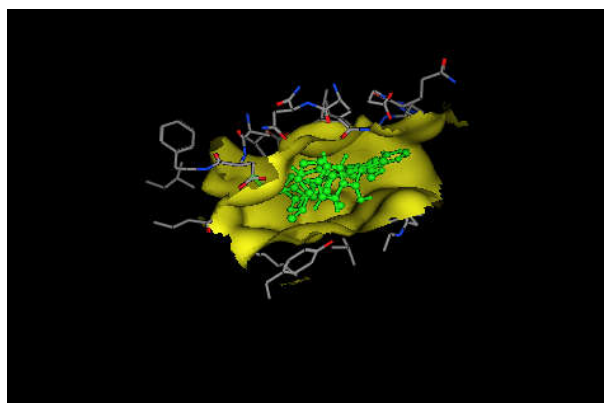

(A)

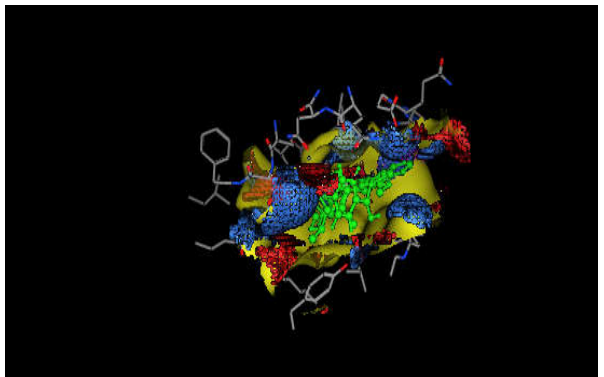

(C)

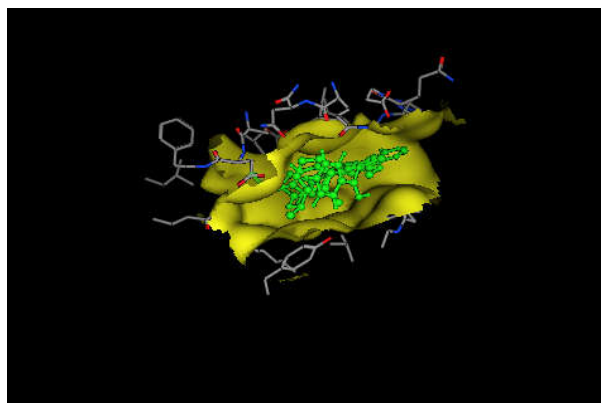

(B)

Figure 4. (A) Docking of compound 13a, (B) ligand interaction of compound 13a, and (C) electrostatic map of compound 13a. Energy of compound $\mathbf{1 3} \mathbf{b}=-24.89$.

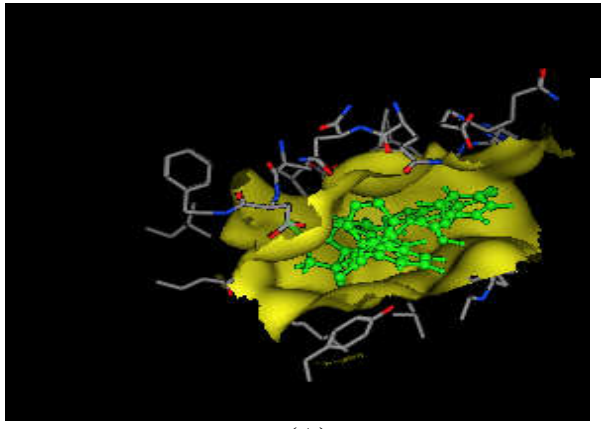

(A)

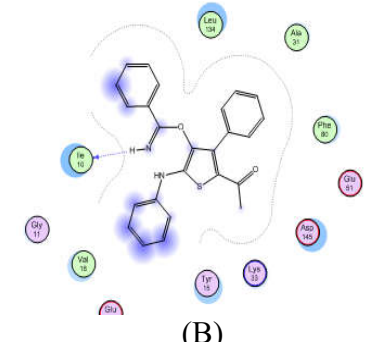

(B) 


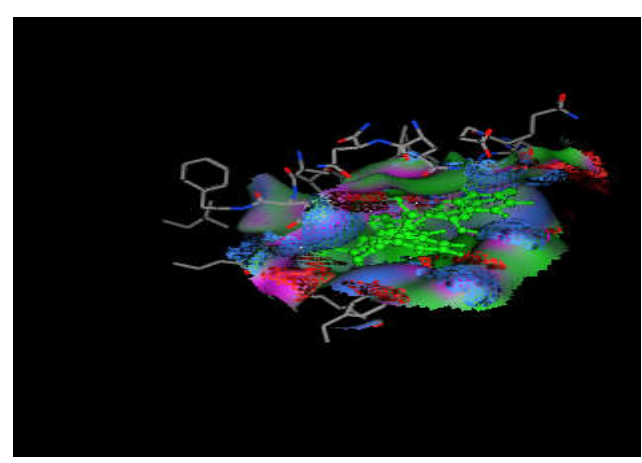

(C)

Figure 5. (A) Energy of compound 16b, (B) ligand interaction of compound 16b, and (C) electrostatic map of compound $\mathbf{1 6} \mathbf{b}$. Energy of compound $\mathbf{1 6 b}=-26.98$.

For Figures 2-5 the blue color indicating the structure of the target molecule and the green color for the amino acid.

\section{CONCLUSION}

The present work, through simple synthetic approaches, led to the development of novel pyridine, thiophene and imidazole derivatives that exhibited remarkable antitumor activities against six tumor cell lines. As new class of heterocyclic compounds, eleven of the obtained new compounds showed remarkable cytotoxicity against most of the six tumor cell lines. Most of the active compounds were devoid of the typical nitrogen and or sulfur feature of heterocyclic derivatives and so the activity could be attributed to some sort of electronegative substituents through the synthesized compounds. In addition c-Met kinase inhibitions for the most active compounds showed that all compounds exhibited inhibitions higher than the reference drug foretinib. The results of the biological screening will encourage future work of heterocyclic compounds derived from $\beta$-diketones.

\section{REFERENCES}

1. Crooks, P.A.; DwoskinmL. P. Contribution of CNS nicotine metabolites to the neuropharmacological effects of nicotine and tobacco smoking. Biochem. Pharm. 1997, 54, 743-753.

2. Jatav, V.; Mishra, P.; Kashaw, S.; Stables, J. P. CNS depressant and anticonvulsant activities of some novel 3-[5-substituted 1,3,4-thiadiazole-2-yl]-2-styryl quinazoline-4(3H)-ones. Eur. J. Med. Chem. 2008, 43, 1945-1954.

3. Warren, B.K.; Knaus, E.E. Pyridine and reduced pyridine analogues of $10 \mathrm{H}-$ pyrido[3,4b] $[1,4]$ benzothiazines with analgesic activity. Eur. J. Med. Chem.1987, 22, 411-415.

4. Mulchin, B.J.; Newton, C.G.; Baty, J.W.; Grasso, C.H.; Martin, W.J.; Walton, M.C.; Dangerfield, E.M.; Plunkett, C.H.; Berridge, M.V.; Harper, J.L.; Timmer, M.S.M.; Stocker, B.L. The anti-cancer, anti-inflammatory and tuberculostatic activities of a series of 6,7substituted-5,8-quinolinequinones. Bioorg. Med. Chem. 2010, 18, 3238-3251.

5. Sangshetti, J.N.; Dharmadhikari, P.P.; Chouthe, R.S.; Fatema, B.; Lad, V.; Karande, V.; Sunil, N.; Darandale, S.N.; Devanand, B.; Shinde, D.B. Microwave assisted nano (ZnO$\left.\mathrm{TiO}_{2}\right)$ catalyzed synthesis of some new 4,5,6,7-tetrahydro-6-((5-substituted-1,3,4-oxadiazol2-yl)methyl)thieno[2,3-c]pyridine as antimicrobial agents. Bioorg. Med. Chem. Lett. 2013, $23,2250-2253$. 
6. Witherington, J.; Bordas, V.; Garland, S.L.; Hickey, D.M.; Ife, R.J.; Liddle, J.; Saunders, M.; Smith, D.G.; Ward, R.W. 5-Aryl-pyrazolo[3,4-b]pyridines: potent inhibitors of glycogen synthase kinase-3 (GSK-3). Bioorg. Med. Chem. Lett. 2003, 13, 1577-1580.

7. Liu, X.H.; Liu, H.F.; Shen, X.; Song, B.A.; Bhadury, P.S.; Zhu, H.L.; Liu, J.X.; Qi, X.B. Synthesis and molecular docking studies of novel 2-chloro-pyridine derivatives containing flavone moieties as potential antitumor agents. Bioorg. Med. Chem. Lett. 2010, 20, 41634167.

8. Ram, S. New benzimidazole carbamates as antifilarial agents. Prog. Med. Chem. 1988, 25, 233-247.

9. Gaba, M.; Singh, S.; Mohan, C. Benzimidazole: an emerging scaffold for analgesic and antiinflammatory agents. Eur. J. Med. Chem. 2014, 76, 494-505.

10. Nevado, J.J.B.; Peñalvo, G.C.; Dorado, R.M.R.; Robledo, V.R. Simultaneous determination of omeprazole and their main metabolites in human urine samples by capillary electrophoresis using electrospray ionization-mass spectrometry detection. J. Pharm. Biomed. Anal. 2014, 92, 211-219.

11. Natsuki Ishizuka, N.; Ken-ichi Matsumura, K.I.; Katsunori Sakai, K.; Masafumi Fujimoto, M.; Shin-ichiMihara, S.I.; Teruo Yamamori, T. Structure-activity relationships of a novel class of endothelin-A receptor antagonists and discovery of potent and selective receptor antagonist, 2-(benzo[1,3]dioxol-5-yl)-6-isopropyloxy-4-(4-methoxyphenyl)-2H-chromene3- carboxylic acid (S-1255). 1. Study on structure-activity relationships and basic structure crucial for ETA antagonism. J. Med. Chem. 2002, 45, 2041-2055.

12. Khoje, A.D.; Charnock, C.; Wan, B.; Franzblau, S.; Gundersen, L.L. Synthesis and antimycobacterial activities of non-purine analogs of 6-aryl-9-benzylpurines: Imidazopyridines, pyrrolopyridines, benzimidazoles, and indoles. Bioorg. Med. Chem. 2011, 19, 3483-3491.

13. Seenaiah, D.; Reddy, P.R.; Reddy, G.M.; Padmaja, A.; Padmavathi, V.; Krishna, N.S. Synthesis, antimicrobial and cytotoxic activities of pyrimidinyl benzoxazole, benzothiazole and benzimidazole. Eur. J. Med. Chem. 2014, 77, 1-7.

14. Yadav, S.; Kumar, P.; Clercq, E.D.; Balzarini, J.; Pannecouque, C.; Dewan, S.K.; Narasimhan, B. 4-[1-(Substituted aryl/alkyl carbonyl)-benzoimidazol-2-yl]-benzenesulfonic acids: Synthesis, antimicrobial activity, QSAR studies, and antiviral evaluation. Eur. J. Med. Chem. 2010, 45, 5985-5997.

15. Zhang, H.Z.; Damu, G.L.V.; Cai, G.X.; Zhou, C.H. Design, synthesis and antimicrobial evaluation of novel benzimidazole type of fluconazole analogues and their synergistic effects with chloromycin, norfloxacin and fluconazole. Eur. J. Med. Chem. 2013, 64, 329344.

16. Dahiya, R.; Pathak, D. Synthetic studies on novel benzimidazolopeptides with antimicrobial, cytotoxic and anthelmintic potential. Eur. J. Med. Chem. 2007, 42, 772-798.

17. Fonseca, T.; Gigante, B.; Marques, M.M.; Gilchrist, T.L.; Clercq, E.D. Synthesis and antiviral evaluation of benzimidazoles, quinoxalines and indoles from dehydroabietic acid. Bioorg. Med. Chem. 2004, 12, 103-112.

18. Herrmann, E.C.; Judith, A.; Herrmann, J.A.; Donald, C.; Delong, D.C. Comparison of the antiviral effects of substituted benzimidazoles and guanidine in vitro and in vivo. Antiviral Res. 1981, 1, 301-314.

19. Sharma, S.; Gangal, S.; Rauf, A. Convenient one-pot synthesis of novel 2-substituted benzimidazoles, tetrahydrobenzimidazoles and imidazoles and evaluation of their in vitro antibacterial and antifungal activities. Eur. J. Med. Chem. 2009, 44, 1751-1757.

20. Shingalapur, R.V.; Hosamani, K.M.; Keri, R.S. Synthesis and evaluation of in vitro antimicrobial and anti-tubercular activity of 2-styryl benzimidazoles. Eur. J. Med. Chem. 2009, 44, 4244-4248. 
21. Pawar, N.S.; Dalal, D.S.; Shimpi, S.R.; Mahulikar, P.P. Studies of antimicrobial activity of $\mathrm{N}$-alkyl and N-acyl 2-(4-thiazolyl)-1H-benzimidazoles. Eur. J. Pharm. Sci. 2004, 21, 115 118.

22. Rashid, M.; Husain, A.; Mishra, R. Synthesis of benzimidazoles bearing oxadiazole nucleus as anticancer agents. Eur. J. Med. Chem. 2012, 54, 855-866.

23. Shaharyar, M.; Abdullah, M.M.; Bakht, M.A.; Majeed, J. Pyrazoline bearing benzimidazoles: Search for anticancer agent. Eur. J. Med. Chem. 2010, 45, 114-119.

24. Abonia, R.; Cortés, E.; Insuasty, B.; Quiroga, J.; Nogueras, M.; Cobo, J. Synthesis of novel 1,2,5-trisubstituted benzimidazoles as potential antitumor agents. Eur. J. Med. Chem. 2011, 46, 4062-4070.

25. Rashid, M.; Husain, A.; Mishra, R.; Anjum, S. Eradication of toxic material by using green chemistry in the development of anticancer drugs. Toxicol. Lett. 2012, 63, 53-56.

26. Badawey, E.A.M.; Kappe, T. Benzimidazole condensed ring systems. XI. Synthesis of some substituted cycloalkyl pyrido[1,2-a]benzimidazoles with anticipated antineoplastic activity. Eur. J. Med. Chem. 1999, 34, 663-667.

27. Huang, S.T.; Hsei, I.J.; Chen, C. Synthesis and anticancer evaluation of bis(benzimidazoles), bis(benzoxazoles), and benzothiazoles. Bioorg. Med. Chem. 2006, 14, 6106-6119.

28. Omar, M.A.; Shaker, Y.M.; Galal, S.A.; Ali, M.M.; Kerwin, S.M.; Li, J.; Tokuda, H. Ramadan, R.A.; El-Diwani, H.I. Synthesis and docking studies of novel antitumor benzimidazoles. Bioorg. Med. Chem. 2012, 20, 6989-7001.

29. Harapanhalli, R.S.; Howel, R.W.; Rao, D.V. Bis-benzimidazole dyes, Hoechst 33258 and Hoechst 33342: Radioiodination, facile purification and subcellular distribution. Nucl. Med. Biol. 1994, 21, 641-647.

30. Singh, S.; Dwarakanath, B.S.; Lazar,T.; Mathew, T.L. DNA ligand Hoechst-33342 enhances UV induced cytotoxicity in human glioma cell lines. J. Photochem. Photobio. B: Biol. 2004, 77, 45-54.

31. Heba, S.A.; Elzahabi, H.A.S. Synthesis, characterization of some benzazoles bearing pyridine moiety: Search for novel anticancer agents. Eur. J. Med. Chem. 2011, 46, 40254035 .

32. Tseng, J.R.; Stuart, D.; Aardalen, K.; Kaplan, A.; Aziz, N.; Hughes, N.P.; Gambhir, S.S. Use of DNA microarray and small animal positron emission tomography in preclinical drug evaluation of RAF265, a novel B-Raf/VEGFR-2 inhibitor. Neoplasia 2011, 13, 266-275.

33. Islam, M.R.; Mohsin, M. Synthesis of isatin, 5-chloroisatin and their $\Delta 2-1,3,4$ oxadiazoline derivatives for comparative cytotoxicity study on brine shrimp. Bangladesh. J. Pharmacol. 2007, 2, 7-12.

34. Bryan, P.; McKibben, B.P.; Craig, H.; Cartwright, C.H.; Arlindo, L.; Castelhano, A.L. Practical synthesis of tetrasubstituted thiophenes for use in compound libraries. Tetrahedron Lett. 1999, 40, 5471-5474.

35. Balamurugan, K.; Perumal, S.; Reddy, A.S.K.; Yogeeswari, P.; Sriram, D. A facile domino protocol for the regioselective synthesis and discovery of novel 2-amino-5-arylthieno[2, 3- $b$ ] thiophenes as antimycobacterial agents. Tetrahedron Lett. 2009, 50, 6191-6195.

36. Calleja, M.C.; Persoone, G. Cyst-based toxicity tests. IV. The potential of ecotoxicological tests for the prediction of acute toxicity in man as evaluated on the first ten chemicals of the MEIC programme. ATLA 1992, 20, 396-405. 